\title{
Diversity of laminar connections linking periarcuate and lateral intraparietal areas depends on cortical structure
}

\author{
M. Medalla ${ }^{1}$ and $\mathrm{H}$. Barbas ${ }^{1,2,3,4}$ \\ ${ }^{1}$ Department of Health Sciences, Boston University, 635 Commonwealth Ave. Room 431, Boston, MA 02215, USA \\ ${ }^{2}$ Program in Neuroscience, Boston University, Boston, MA, USA \\ ${ }^{3}$ NEPRC, Harvard Medical School, Boston, MA, USA \\ ${ }^{4}$ Department of Anatomy and Neurobiology, Boston University Medical School, Boston, MA, USA
}

Keywords: 7a, areas LIPv, FEF, lateral prefrontal, LIPd, Macaca mulatta, quantitative architecture

\begin{abstract}
Lateral prefrontal and intraparietal cortices have strong connectional and functional associations but it is unclear how their common visuomotor, perceptual and working memory functions arise. The hierarchical scheme of cortical processing assumes that prefrontal cortex issues 'feedback' projections to parietal cortex. However, the architectonic heterogeneity of these cortices raises the question of whether distinct areas have laminar-specific interconnections underlying their complex functional relationship. Using quantitative procedures, we showed that laminar-specific connections between distinct prefrontal (areas 46 and 8) and lateral intraparietal (LIPV, LIPd and 7a) areas in Macaca mulatta, studied with neural tracers, varied systematically according to rules determined by the laminar architecture of the linked areas. We found that axons from areas 46 and rostral 8 terminated heavily in layers I-III of all intraparietal areas, as did caudal area 8 to area LIPv, suggesting 'feedback' communication. However, contrary to previous assumptions, axons from caudal area 8 terminated mostly in layers IV-V of LIPd and 7a, suggesting 'feedforward' communication. These laminar patterns of connections were highly correlated with consistent differences in neuronal density between linked areas. When neuronal density in a prefrontal origin was lower than in the intraparietal destination, most terminations were found in layer I with a concomitant decrease in layer IV. The opposite occurred when the prefrontal origin had a higher neuronal density than the target. These findings indicate that the neuronal density of linked areas can reliably predict their laminar connections and may form the basis of understanding the functional complexity of prefrontal-intraparietal interactions in cognition.
\end{abstract}

\section{Introduction}

Periarcuate prefrontal and posterior parietal areas are associated with oculomotor functions linked to cognitive operations of working memory, attention and spatial perception (reviewed in Lynch, 1980; Hyvärinen, 1982; Fuster, 1984; Colby \& Goldberg, 1999; Tehovnik et al., 2000). Lesions of these areas adversely affect these cognitive operations, manifested as the neurologic symptom of neglect (reviewed in Mesulam, 1999). The common functions of periarcuate and parietal cortices probably depend on their strong interconnections (Barbas \& Mesulam, 1981; Petrides \& Pandya, 1984; Andersen et al., 1985; Cavada \& Goldman-Rakic, 1989b; Stanton et al., 1995) and overlapping connections with visual association and premotor areas (Seltzer \& Pandya, 1980; Barbas \& Pandya, 1987; Barbas, 1988; Andersen et al., 1990a; Blatt et al., 1990; Baizer et al., 1991; Cusick et al., 1995; Schall et al., 1995). Stimulation of periarcuate and lateral intraparietal regions evokes eye movements (Robinson \& Fuchs, 1969; Shibutani et al., 1984) and neurons in these cortices have stimulus-driven and saccade-related responses during visuomotor working memory tasks (Fuster, 1973; Kubota et al., 1974; Niki \& Watanabe, 1976; Wurtz \& Mohler, 1976; Lynch et al., 1977; Bushnell et al., 1981; Bruce \& Goldberg, 1985; Andersen et al., 1987; Colby et al., 1996).

Correspondence: Dr Helen Barbas, ${ }^{1}$ Department of Health Sciences, as above. E-mail: barbas@bu.edu

Received 27 July 2005, revised 17 October 2005, accepted 29 October 2005
Prefrontal-parietal interactions are thought to mediate sensorymotor transformation and decision-making for cognitive processing (Quintana \& Fuster, 1993; Quaia et al., 1998; Shadlen \& Newsome, 2001; Williams et al., 2003). However, it is not clear where decisionrelated activity arises because periarcuate and intraparietal areas have similar activation patterns during cognitive tasks (Chafee \& GoldmanRakic, 1998; Wurtz et al., 2001). Moreover, periarcuate areas 46 and 8 are architectonically and functionally distinct, as are the ventral and dorsal divisions of the lateral intraparietal area (LIP; LIPv and LIPd, respectively) and area 7a (Seltzer \& Pandya, 1980; Andersen et al., 1990a; reviewed in Barbas, 1992), and may have unique roles in cognition.

Laminar patterns of connections provide important clues on corticocortical interactions. Prefrontally directed intraparietal neurons originate mostly in layer III (Barbas \& Mesulam, 1981; Andersen et al., 1985; Schall et al., 1995), resembling 'feedforward' communication. The reciprocal projections arise from prefrontal layers III, V and VI and terminate in a bilaminar 'feedback' pattern in intraparietal cortex, or a columnar pattern, akin to 'lateral' connections (Andersen et al., 1990a; Schall et al., 1995; Stanton et al., 1995). However, previous studies relied largely on qualitative or global quantitative descriptions and it is not clear whether the laminar distribution of specific prefrontal-intraparietal pathways varies systematically.

The heterogeneity of periarcuate and intraparietal areas raises the question of whether distinct areas have laminar-specific connections that may account for their complex functional interactions. This 
question emerged from evidence that the structural relationship of linked areas can predict their laminar interconnections (Barbas \& Rempel-Clower, 1997; Rempel-Clower \& Barbas, 2000). Using quantitative methods to investigate architecture and connections, we provide novel evidence that the laminar connections of areas 46 and 8 in intraparietal areas LIPv, LIPd and 7a vary consistently according to rules based on their structural relationship. The consistent pattern of connections may underlie specific aspects of communication between these cortices in cognitive operations.

\section{Materials and methods}

\section{Surgical procedures}

Experiments were conducted on nine adult rhesus monkeys (Macaca mulatta) according to the NIH guide for the Care and Use of Laboratory Animals [DHEW Publication no. (NIH) 80-22, revised 1987, Office of Science and Health Reports, DRR/NIH, Bethesda, MD, USA]. The monkeys were anaesthetized with ketamine hydrochloride $(10 \mathrm{mg} / \mathrm{kg}$, intramuscularly) followed either by sodium pentobarbital (to effect) or by isoflurane until a surgical level of anaesthesia was accomplished; this was maintained throughout surgery. The monkeys were placed in a stereotaxic apparatus and a small region of the prefrontal cortex was exposed. Surgery was performed under aseptic conditions and heart rate, muscle tone, respiration and pupillary dilatation were closely monitored.

\section{Injection of neural tracers}

In each of three animals (cases $\mathrm{MBH}, \mathrm{AC}$ and $\mathrm{AD}$ ), a small site of the periarcuate cortex was injected with the bidirectional tracer horseradish peroxidase conjugated to wheatgerm agglutinin (HRP-WGA; 8\% solution, total volume $0.1 \mu \mathrm{L}$; Sigma-Aldrich, St Louis, MO, USA). The fluorescent dyes fluororuby (FR; case BFr; $10 \%$ solution, total volume $2 \mu \mathrm{L}$; dextran tetramethylrhodamine, MW 3000; Molecular Probes, Eugene, OR, USA) and fluoroemerald (FE; case BFe; 10\% solution, total volume $3 \mu \mathrm{L}$; dextran fluorescein, MW 10000 ; Molecular Probes) were injected in one animal. In each case, the tracer was injected in two penetrations separated by $\sim 0.5 \mathrm{~mm}$, at a depth of 1.2-1.6 mm below the pial surface using a 5- $\mu \mathrm{L}$ microsyringe (Hamilton, Reno, NV, USA) mounted on a microdrive. The microsyringe was left in place for a period of 10-15 min to allow local diffusion of the dye at the injection site and prevent upward suction of the dye upon retraction of the needle.

\section{Perfusion and tissue processing}

In all cases, the animals were given a lethal dose of anaesthetic (sodium pentobarbital, $>50 \mathrm{mg} / \mathrm{kg}$, to effect) and perfused through the heart. In cases with injection of HRP-WGA, the survival period was 2 days and perfusion was initiated with saline followed by $2 \mathrm{~L}$ of fixative $[1.25 \%$ glutaraldehyde, $1 \%$ paraformaldehyde in $0.1 \mathrm{M}$ phosphate buffer (PB), pH 7.4] and then $2 \mathrm{~L}$ of cold $\left(4^{\circ} \mathrm{C}\right) \mathrm{PB}$ $(0.1 \mathrm{M}, \mathrm{pH} 7.4)$. The brain was then removed from the skull, photographed and placed in $10 \%$ glycerol phosphate buffer $(10 \%$ glycerol, 2\% dimethyl sulfoxide; Sigma; in $0.1 \mathrm{M} \mathrm{PB}, \mathrm{pH} 7.4$ ) for 1 day and then in $20 \%$ glycerol phosphate buffer for two additional days. In the experiment with injections of fluorescent dyes, the survival period was 19 days and the animal was perfused with $2 \mathrm{~L}$ of fixative (4\% paraformaldehyde in $0.1 \mathrm{M} \mathrm{PB}$ with $0.9 \% \mathrm{NaCl}, \mathrm{pH} 7.4$ ). The brain was removed from the skull, photographed with a CCD camera and cryoprotected in sucrose solutions of increasing concentrations $(10-30 \%$ in $0.1 \mathrm{M} \mathrm{PB}$ with $0.9 \% \mathrm{NaCl}, \mathrm{pH} 7.4$, and $0.05 \%$ sodium azide; Sigma).

Brain tissue from five rhesus monkeys was used for immunocytochemical analysis of quantitative architectonic features in lateral intraparietal and prefrontal cortices. These animals were perfused with the same fixative and the brains were cryoprotected as in the fluorescent tracer case.

All brains were frozen in $-75{ }^{\circ} \mathrm{C}$ isopentane (Rosene et al., 1986) and cut on a freezing microtome in the coronal plane at 40 or $50 \mu \mathrm{m}$ to produce 10 series. In the HRP-WGA experiments, one series from each case was treated to visualize HRP (Mesulam et al., 1980). The tissue was mounted, dried and counterstained with neutral red (Fisher Scientific International Inc., Hampton, NH, USA). In the experiment with injections of fluorescent tracers, brain sections were mounted on glass slides immediately after cutting and every other section was stained with thionin (Fisher Scientific) to delineate areal and laminar boundaries.

\section{Immunocytochemical procedures}

\section{Immunocytochemistry (brightfield)}

We used immunocytochemical staining for quantitative analysis of neuronal nuclei-specific antibody (NeuN), SMI-32, which labels the nonphosphorylated neurofilament $\mathrm{H}$ found in a subclass of projection neurons, and the calcium-binding proteins parvalbumin (PV) and calbindin (CB), which primarily label two distinct neurochemical classes of interneurons in the cortex (Hendry et al., 1989; Campbell et al., 1991; Condé et al., 1994; Gabbott \& Bacon, 1996; Glezer et al., 1998; DeFelipe et al., 1999; Dombrowski et al., 2001; Barbas et al., 2005b). In the experiment with injections of fluorescent dyes (cases BFe and BFr), we converted four adjacent series (two series per tracer) to brightfield label using antibodies for either FE or FR.

Free-floating sections were washed $(3 \times 10 \mathrm{~min}$, under slow agitation) with $0.01 \mathrm{M}$ phosphate-buffered saline (PBS; pH 7.4) and preblocked for $1 \mathrm{~h}$ with $10 \%$ normal goat serum (NGS; Vector Laboratories, Burlingame, CA, USA) with $0.2 \%$ Triton-X (Sigma) in $0.01 \mathrm{M}$ PBS. After rinsing, the tissue was incubated overnight in a solution containing $1 \%$ NGS, $0.1 \%$ Triton-X in $0.01 \mathrm{M}$ PBS, and the primary antibody for either $\mathrm{NeuN}(1: 200$, mouse monoclonal; Chemicon International Inc., Temecula, CA, USA), SMI-32 (1 : 5000, mouse monoclonal; Sternberger Monoclonals, Lutherville, MD, USA), PV (1 : 2000, mouse monoclonal; Chemicon), CB (1:2000, mouse monoclonal; Accurate Chemicals and Scientific Corp., Westbury, NY, USA), FE (1 : 800, rabbit polyclonal; Molecular Probes), or FR (1 : 800, rabbit polyclonal; Molecular Probes). Sections were then rinsed in PBS and incubated for $3 \mathrm{~h}$ in biotinylated secondary antibody solution $(1: 200$, with $1 \%$ NGS and $0.1 \%$ Triton-X in 0.01 M PBS; goat antimouse IgG for NeuN, SMI-32, CB and PV; goat antirabbit IgG for FE and FR; Vector Laboratories). After incubation the sections were washed in PBS and processed for immunocytochemistry according to instructions in the avidin-biotin reagent kit (with $0.1 \%$ Triton-X in $0.01 \mathrm{M}$ PBS; Vectastain PK 4000 AvidinBiotin Peroxidase Kit, Vector Laboratories). Tissue was washed in PBS and processed for 1-2 min for immunoperoxidase reaction (3, $3^{\prime}$ diaminobenzidine tetrahydrochloride, DAB-plus kit; Zymed Laboratories Inc., South San Francisco, CA, USA). Sections were placed in $\mathrm{PB}(0.1 \mathrm{M}, \mathrm{pH} 7.4)$ for a final rinse, mounted and dried. Every other section was counterstained with thionin, and sections were coverslipped with permount. 
Double immunocytochemistry to simultaneously visualize tracers and $C B$ or $P V$

We employed immunocytochemical procedures using fluorescent probes to visualize the distribution of $\mathrm{CB}$ or $\mathrm{PV}$ in lateral intraparietal areas along with fluorescent tracers (FE). Matched series of sections were incubated in primary antibody for either CB or PV (as described above). The tissue was then placed overnight in goat antimouse IgG conjugated with the fluorescent probe cyanoindocarbocyanine (Cy3, $550-570 \mathrm{~nm}$, Chemicon; $1: 800$, with $1 \%$ NGS and $0.1 \%$ Triton-X in $0.01 \mathrm{M}$ PBS) and rinsed in PB.

To visualize FE-labelled axonal boutons from prefrontal cortices and CB or PV simultaneously in intraparietal areas using brightfield illumination, we used two immunocytochemical methods to avoid cross-reaction, and two separate peroxidase precipitation reactions to yield distinct staining. We used the peroxidase-antiperoxidase (PAP) method and $\mathrm{DAB}$ reaction to view FE, and the avidin-biotin method using the VIP substrate (Vector VIP Substrate Kit, Vector Laboratories) to visualize $\mathrm{CB}$ or PV. We first incubated the tissue in the primary antibody for FE (as described above). Sections were rinsed in PBS, followed by a 2-h incubation in nonbiotinylated secondary antibody ( $1: 200$, with $1 \%$ NGS and $0.1 \%$ Triton-X in $0.01 \mathrm{M}$ PBS; goat antirabbit IgG, Sternberger Monoclonals), which does not crossreact with the avidin-biotin complex. After washing in PBS, we incubated the tissue in the PAP tertiary antibody for $1 \mathrm{~h}(1: 200$, with $1 \%$ NGS and $0.1 \%$ Triton-X in $0.01 \mathrm{M}$ PBS; activity-select rabbit PAP, Sternberger Monoclonals) and processed with DAB (as above). Tissue was then incubated in the primary antibody for CB or PV, followed by a biotinylated secondary antibody and then the avidin-biotin solution (as above). Sections were washed in PBS and placed for $\sim 10 \mathrm{~s}$ in VIP. The DAB reaction product (brown) was clearly distinguishable from the VIP reaction product (purple).

\section{Data analysis}

\section{Stereologic procedures}

We employed stereologic procedures to estimate the density of different neuronal populations (NeuN, SMI-32, CB, PV) in the lateral intraparietal areas. We also estimated the density of neurons, marked by NeuN, in distinct subdivisions of the periarcuate prefrontal cortex, which were matched with the respective injection sites. We used a semiautomated commercial system (StereoInvestigator; MicroBrightField Inc., Williston, VT, USA) and viewed brain tissue at $600 \times$ magnification under oil immersion (Olympus BX51). We placed areal and laminar boundaries from Nissl-stained coronal sections, based on current observations and previous architectonic maps (Seltzer \& Pandya, 1980; Andersen et al., 1985; Barbas \& Pandya, 1989; Andersen et al., 1990a; Lewis \& Van Essen, 2000b). Separate counts were made for the superficial layers (layers I-III) and the deep layers (layers IV-VI). For sections stained with SMI-32, layers I and IV were not included in the counting area because staining was absent in these layers. For sections stained with NeuN, where laminar boundaries are distinct, neurons in layers II-III, IV and V-VI were counted separately.

The counting parameters included the coefficient of error (set to $<10 \%$ ), target cell counts, section interval, counting frame size, grid size, section thickness and guard zone size (for reviews see Gundersen et al., 1988; Howard \& Reed, 1998; Schmitz \& Hof, 2005). Counting using an optical disector was restricted to a fraction of the tissue thickness and included a guard zone to avoid error due to cell plucking or cell splitting during tissue sectioning. Section thickness was $50 \mu \mathrm{m}$ after cutting, which shrank to $15 \mu \mathrm{m}$ after processing and mounting on gelatin-coated slides. The guard zone was set at $2 \mu \mathrm{m}$ for the top and bottom of the tissue section, leaving $11 \mu \mathrm{m}$ in the counting brick. After pilot study, the counting frame area was set at $150 \times 150 \mu \mathrm{m}$.

The density of cells per $\mathrm{mm}^{3}$ was obtained by dividing the estimated number of neurons over the volume of the region examined. The mean and SEM regional and laminar densities for each neurochemical class of neurons were obtained from three animals. We normalized the laminar densities for statistical analyses by obtaining the percentage density of cells in the superficial and deep layers.

\section{Mapping of projection neurons}

Coronal sections in one series (representing 1 in 10 sections) ipsilateral to the injection site were viewed under brightfield or fluorescence illumination $(200 \times)$ to map retrogradely labelled neurons within the lateral intraparietal cortex. In the experiment with injections of fluorescent tracers, projection neurons were viewed and mapped directly from one series of sections mounted on glass slides immediately after cutting and from two series processed for immunocytochemistry to view label under brightfield illumination. In some cases, labelled neurons were mapped using a digital plotter (Hewlett Packard 7475A), which was electronically coupled to the stage of the microscope (Nikon Optiphot) and to a PC computer, using software designed in our laboratory, ensuring that each labelled neuron was counted only once, as described previously (e.g. Barbas \& De Olmos, 1990). In some cases, we mapped labelled neurons using a microscope (Olympus BX51) coupled to a computer and a commercial software system (Neurolucida, MicroBrightField Inc.). In all cases, we conducted exhaustive sampling through the lateral intraparietal cortex and counted labelled neurons by area and layer for each series of sections.

\section{Analysis of anterograde label}

For cases with fluorescent tracers processed for immunocytochemistry to view label under brightfield illumination $(400 \times)$, we conducted exhaustive quantitative mapping of labelled boutons from two adjacent series by area and layer using the Neurolucida tracing system (MicroBrightField Inc.). We computed the volume of each layer in each intraparietal area by multiplying the total laminar surface area by the total thickness of sections plotted, and expressed bouton density per $\mathrm{mm}^{3}$.

Because anterograde HRP-WGA labelling does not appear as discrete boutons, we measured the density of axonal terminations using optical density with the aid of an image analysis system (MetaMorph v4.1; Universal Imaging System Corp., Downingtown, PA, USA), as described previously (Barbas \& Rempel-Clower, 1997). Each coronal section in one series was viewed under darkfield illumination $(100 \times)$ using a microscope (Olympus BX51) equipped with a CCD camera (Dage-MTI, Michigan City, IN, USA) and a fibreoptic illuminator that yields uniform lighting conditions (Optical Analysis Corp., Nashua, NH, USA). Background intensity levels were obtained by an initial density measure in each section in a region with no anterograde label, and were subtracted from subsequent optical density measurements from each layer in each area. This method is highly reliable and has shown high correlation among independent users (Barbas \& Rempel-Clower, 1997; Rempel-Clower \& Barbas, 1998; Barbas et al., 1999).

\section{Normalized density of projection neurons and axonal terminations for regional and laminar comparisons}

We obtained relative regional density data by expressing the number of labelled neurons or the density of labelled terminations in each area 
as a percentage of the total in the lateral intraparietal cortex. We expressed relative laminar densities as the percentage in the upper (layers II-III) and deep (layers V-VI) layers (for projection neurons), or the percentage in layers I, II-III, IV and V-VI (for axonal terminations). Only areas with significant label ( $>20$ neurons or $>50$ boutons) were used for regional and laminar comparisons. For optical density, the lower limit was defined as values above background levels, which is equivalent to $\sim 17 \%$ of the densest label and corresponds to $\sim 50$ boutons in cases with discrete labelled varicosities, as described previously (Barbas et al., 2005b).

\section{Two-dimensional and three-dimensional reconstructions of connections}

In each case, we mapped the rostrocaudal level of each coronal section with label in the intraparietal cortex on the two-dimensional lateral surface of the brain, using $0.5-\mathrm{mm}$ intervals. The depth of the label in the intraparietal sulcus was estimated from coronal photomicrographs. The densities of retrograde and anterograde label in each intraparietal area were represented with distinct symbols (for neuronal and bouton counts) or in pseudocolour (for optical density).

To compare the topography and laminar distribution of connections across cases on a reference brain, we made a three-dimensional (3-D) reconstruction of the lateral bank of the intraparietal sulcus by adapting the software Reconstruct (Fiala, 2005). We scanned and aligned all Nissl-stained coronal sections spanning the rostrocaudal extent of the intraparietal sulcus from one series in one of three cases (cases $\mathrm{MBH}, \mathrm{BFe}$ and $\mathrm{AD}$ ). We reconstructed the 3-D surface of the lateral intraparietal bank, delineated the boundaries of layers I and IV and used this case as reference. We then matched and aligned sections with label from all three cases with their corresponding sections in the reference series. We traced patches of label from the coronal sections and mapped them on the reconstructed 3-D surface of the lateral intraparietal bank, superimposing the connections from three distinct prefrontal injections. The resulting 3-D map shows the relative rostrocaudal extent, depth and laminar distribution of different prefrontal connections in the intraparietal cortex for direct comparison.

\section{Statistical analyses}

We used one-way ANOvA to test for differences among areas and nonparametric multidimensional scaling (NMDS) to determine global similarities and differences among lateral intraparietal areas based on multiple architectonic parameters (normalized density of $\mathrm{NeuN}^{+}$, SMI$32^{+}, \mathrm{CB}^{+}$and $\mathrm{PV}^{+}$neurons in supragranular layers), or the entire complement of their connectivity patterns (percentage of projection neurons and axonal terminations in the upper layers). NMDS analysis was conducted in Statistica (v.6 for Windows; StatSoft Inc., Tulsa, OK, USA), employing squared data (dis)similarity matrices derived from either normalized laminar cell density or connectivity profiles by Pearson's correlation, as described previously (Dombrowski et al., 2001). NMDS considers all parameters within the multidimensional scale and plots cortical areas as points in a chosen low-dimensional space (two-dimensional, in this case) based on pairwise correlation (dis)similarities between areas. The relative proximity between the points in the NMDS space represents their relative similarity.

To investigate the relationship between laminar connection patterns and neuronal density (ND) difference of linked prefrontal and intraparietal areas, we conducted correlation (Pearson's $r$ ) and linear regression (SPSS v.10.1 and SigmaPlot 2001 v.7.0 for Windows; SPSS Inc., Chicago, IL, USA). The neuronal density difference for each pair of linked areas was obtained by subtracting the mean density of neurons positive for $\mathrm{NeuN}$ in the target area from the density in its corresponding area of origin $\left(\mathrm{ND}_{\text {origin }}-\mathrm{ND}_{\text {target }}\right)$. To test whether laminar connection patterns change with respect to neuronal density differences between linked areas, we took the percentage of terminations (in either layer I or layer IV), or projection neurons (in layers IIIII or $\mathrm{V}-\mathrm{VI}$ ), as the dependent variable $(Y)$ and plotted it against neuronal density difference, taken as the independent variable $(X)$.

We also used an alternative approach to determine the relationship between laminar termination patterns and structure, using the ordinal structural type difference $(\Delta)$ of the linked areas, according to the structural model for laminar connections proposed in previous studies (e.g. Barbas \& Rempel-Clower, 1997). In this scheme, a rating (1-5) was previously assigned to areas, reflecting the lowest (1) to the highest (5) number of distinguishable layers and overall neuronal density. Because periarcuate prefrontal and intraparietal areas in the present study are all eulaminate areas with six layers, belonging to structural level 5 , we used a finer scale $(0.25$ increments $)$ to rate the areas as subtypes of level 4-5. Areas with the highest neuronal density were assigned a rating of 5 (areas LIPv and caudal 8), followed by areas rated 4.75 (areas LIPd and intermediate 8, caudal part), 4.5 (areas 7a and intermediate 8 , rostral part) and 4.25 (areas 46 and rostral 8 ). We then employed correlation and linear regression analyses by plotting $\Delta$ (rating of prefrontal origin - rating of intraparietal target) against the percentage of terminations in layer I or layer IV.

\section{Photography}

Photomicrographs of labelled boutons, fibers and neurons in lateral intraparietal areas were captured with a CCD camera mounted on a microscope (Olympus BX51) connected to a PC using either MetaMorph (Universal Imaging Corp.) or Neurolucida (MicroBrightField Inc.) image analysis systems. In cases with HRP injections, sections were viewed and photographed under darkfield illumination. To show the pattern of label throughout the entire lateral bank of the intraparietal sulcus, we obtained photomicrographs at low magnification (40×) using software that automatically captures and joins adjacent parts of the area of interest at high resolution (Virtual Slice, Neurolucida, MicroBrightField Inc.).

To photograph simultaneously FE-labelled boutons and calbindin$\left(\mathrm{CB}^{+}\right)$or parvalbumin $\left(\mathrm{PV}^{+}\right)$-positive neurons, we acquired image stacks of several focal planes in each area of interest and created pictures of 50- $\mu \mathrm{m}$-thick sections focused throughout their $z$-axis extent using ImageJ (v.1.32j for Windows; Wayne Rasband, NIH, Bethesda, MD, USA; http://rsb.info.nih.gov/ij/, 1997-2005). This procedure was also used to capture images of $\mathrm{PV}^{+}$or $\mathrm{CB}^{+}$neurons (in pseudocolour) in lateral intraparietal areas stained with fluorescent probes. Stacked images from adjacent serial sections were superimposed to highlight the differential distribution of labelled neurons. Figures were prepared with Adobe Photoshop (v.6.0 for Windows, Adobe Systems Inc., San Jose, CA, USA) and overall brightness and contrast were adjusted without retouching.

\section{Results \\ Overview of the architecture of lateral intraparietal areas}

We delineated architectonic boundaries of the posterior lateral intraparietal bank, from series of sections stained for Nissl, based on maps from previous architectonic, physiologic and connectional studies (Seltzer \& Pandya, 1980; Andersen et al., 1985; Cavada \& Goldman-Rakic, 1989a; Andersen et al., 1990a; Lewis \& Van Essen, 2000 b) and observations from the current study (Fig. 1A-C). Area LIP 


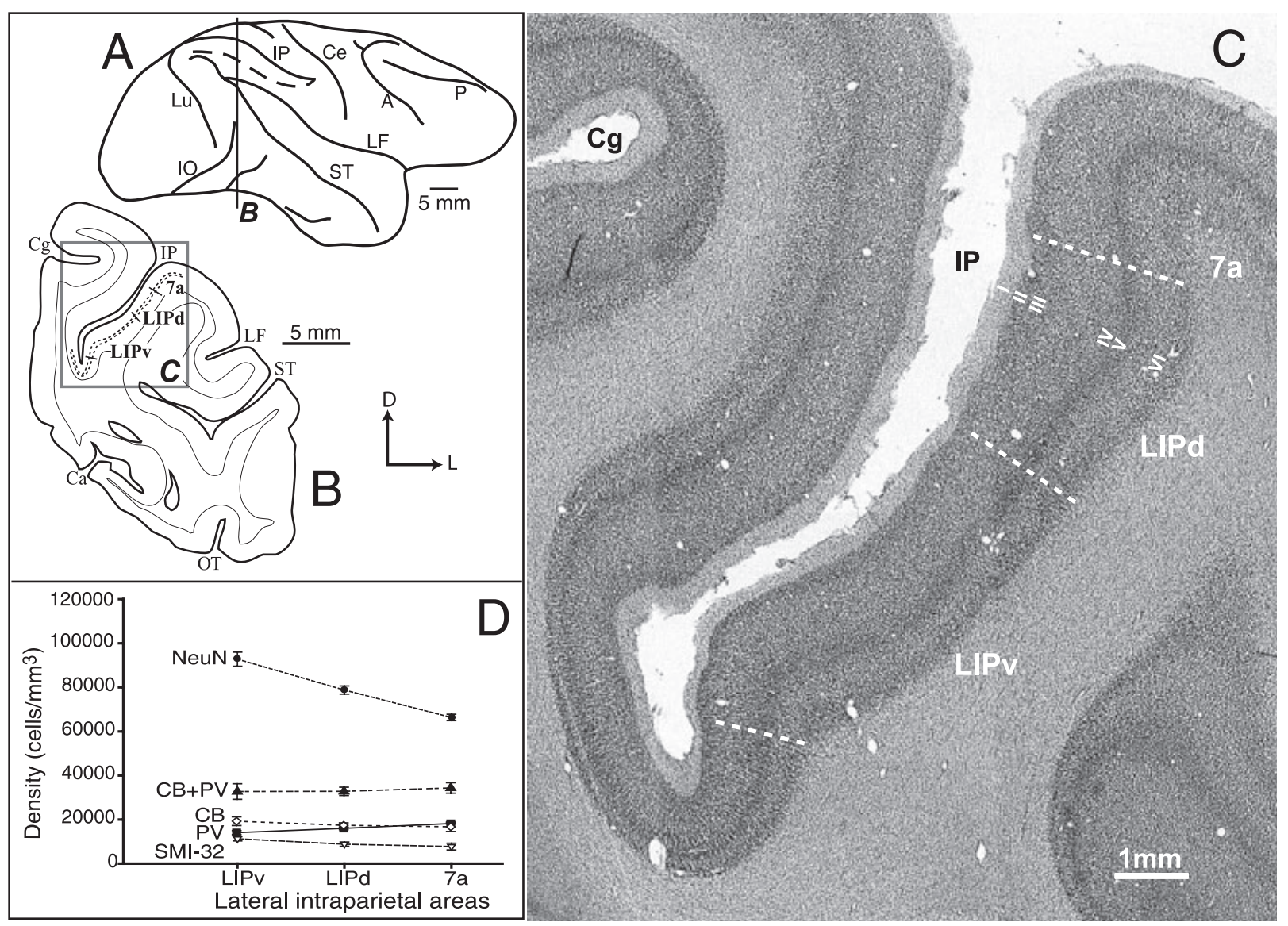

FIG. 1. Architectonic differences in lateral intraparietal cortex. (A) Lateral surface of the brain, showing the anteroposterior location of B. A, arcuate sulcus; Ca, calcarine sulcus; Ce, central sulcus; Cg, cingulate sulcus; IO, inferior occipital sulcus; IP, intraparietal sulcus; LF lateral fissure; Lu, lunate sulcus; OT, occipitotemporal sulcus; P, principal sulcus; ST, superior temporal sulcus. (B) Coronal section through the lateral intraparietal cortex showing the location of section in C. Dotted lines mark boundaries of layer IV. D, dorsal; L, lateral axes. (C) Nissl-stained section showing the areal boundaries in the lateral bank of the intraparietal sulcus (LIPv, LIPd and 7a); laminar labels are placed at the beginning of each layer. (D) A summary of the regional densities of neurons expressing NeuN, SMI-32, PV, CB and CB + PV combined, in lateral intraparietal areas.

of Andersen et al. (1985) corresponds to architectonic area POa of Seltzer \& Pandya (1980) and is buried within the depths of the lateral bank of the intraparietal sulcus; it is subdivided into a ventromedial (LIPv/POa-i) and a dorsolateral (LIPd/POa-e) part. Area LIPv covers approximately the deepest half of the lateral intraparietal bank (Fig. 1B). It has a distinct cortical layer II that forms a sharp boundary with layer I (Fig. 1C). Layer III is broad and has delineated sublayers, with prominent pyramidal neurons in layer IIIc. Layer IV is broad and is interrupted by protruding neurons from the deep part of layer III and the upper part of layer V. Layer V is well differentiated from layer VI. Area LIPd is located more superficially, extending outward from the middle of the lateral intraparietal bank, covering about a fourth to a third of the bank (Fig. 1B). Compared to LIPv, the boundary of layer II is not as sharp, the large pyramidal neurons in layer III are not as prominent and layer IV is relatively narrow. Layers V and VI are broad, but the boundary between them is not as sharp as in area LIPv (Fig. 1C). The cortex adjacent to area LIPd is area 7a of Vogt \& Vogt (1919) (reviewed by Cavada \& GoldmanRakic, 1989a) and corresponds to area PG of Von Bonin \& Bailey (1947) and Seltzer \& Pandya (1980). Area 7a extends from the outermost quarter of the lateral intraparietal bank to the surface of the inferior parietal lobule (Fig. 1B) and continues posteriorly on the surface of the hemisphere reaching approximately the shoulder of the superior temporal sulcus. It has a distinct columnar appearance, with neurons in layers II-III stacked vertically, forming prominent radial arrays (Fig. 1C). Compared to areas LIPd and LIPv, layers IV, V and VI are broad, and the boundary between layers V and VI is not as prominent. Its pyramidal neurons in layer IIIc are large and prominent and its cortical layer II is less distinct than in area LIPd.

\section{Quantitative architecture of the lateral intraparietal areas}

We investigated the quantitative architecture of distinct lateral intraparietal areas using stereologic procedures to estimate the density of specific neuronal populations expressing NeuN, SMI-32, PV and CB.

\section{Neuronal and SMI-32 density varied in parallel across lateral intraparietal areas}

Neurons positive for $\mathrm{NeuN}\left(\mathrm{NeuN}^{+}\right)$showed a significant decrease in overall density along a mediolateral direction (ANOvA, $P=0.003$ ), with LIPv having the highest density and area 7 a the lowest density (Figs 1D and 2A). This decrease was found in both supragranular (IIIII, $P=0.05)$ and infragranular (V-VI, $P=0.04$ ) layers of all intraparietal areas, but the supragranular layers consistently had higher neuronal densities than the infragranular layers $(P=0.04$; Fig. $2 \mathrm{~B})$. We saw the same trend for the overall density of SMI- $32^{+}$neurons, 

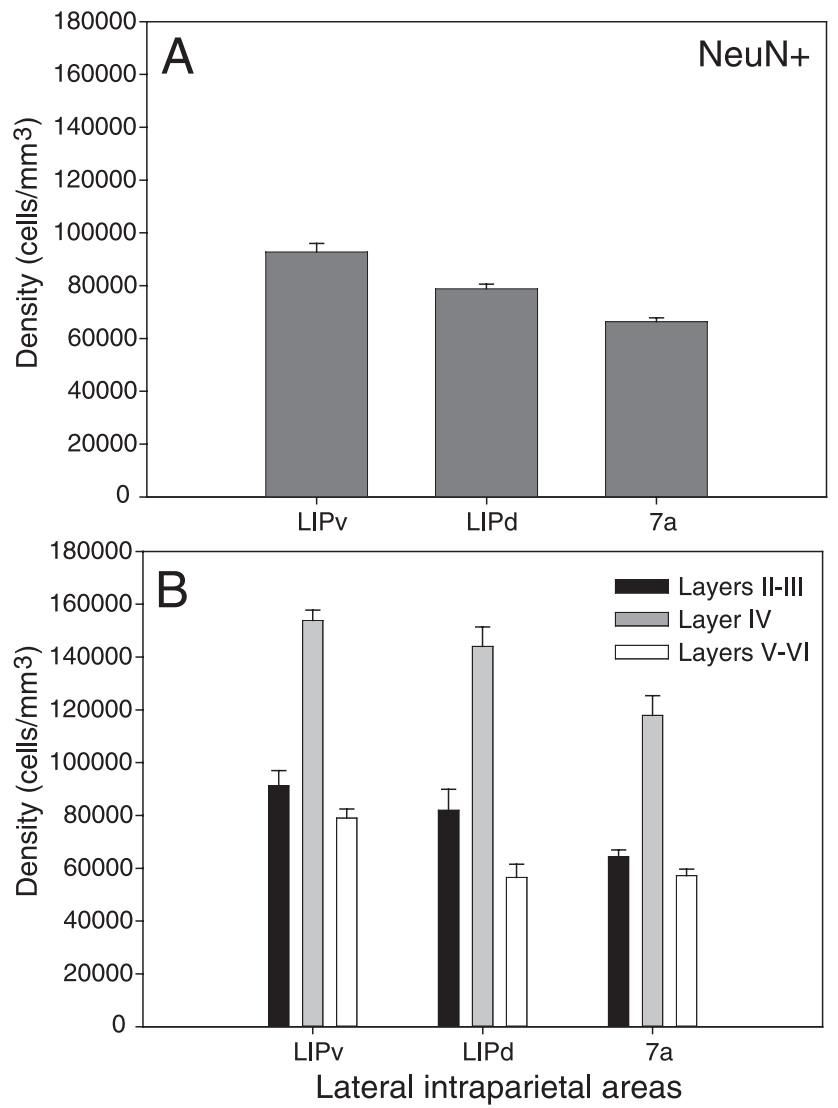

FIG. 2. Density of neurons labelled with NeuN in lateral intraparietal areas. (A) Regional density; (B) laminar density.

significantly correlating with the density of neurons marked by $\mathrm{NeuN}$ (Pearson's $r=0.714, P=0.031$; Figs $1 \mathrm{D}$ and 3A). The density of SMI- $32^{+}$neurons varied significantly only in the supragranular layers $(P=0.04$; Fig. 3B).

\section{$P V^{+}$and $\mathrm{CB}^{+}$neurons varied in opposite directions in lateral intraparietal areas}

The overall distribution of $\mathrm{CB}^{+}$and $\mathrm{PV}^{+}$neurons varied along opposite trends across intraparietal areas (Figs 1D and 4A), revealing a complementary regional distribution, as noted in other cortices (e.g. Hendry et al., 1989; Condé et al., 1994; Kondo et al., 1994; Gabbott \& Bacon, 1996; Glezer et al., 1998; DeFelipe et al., 1999; Dombrowski et al., 2001; Barbas et al., 2005b). In area LIPv, the density of $\mathrm{CB}^{+}$cells was higher than in area LIPd or area 7a, specifically due to an increase in the superficial layers $(P=0.01$; Fig. 4B). In contrast, the overall density of $\mathrm{PV}^{+}$neurons in area LIPV was lower than for $\mathrm{CB}^{+}$neurons, while in areas LIPd and 7a there was a small, but significant, increase in the overall density of $\mathrm{PV}^{+}$neurons $(P=0.01$; Fig. $4 \mathrm{~A})$, due to a graded increase in the superficial layers $(P=0.03$; Fig. 4C).

Although $\mathrm{CB}^{+}$and $\mathrm{PV}^{+}$neurons were more prevalent in the upper layers (I-III), the proportion of $\mathrm{CB}^{+}$neurons in the superficial layers was higher than $\mathrm{PV}^{+}$neurons for all intraparietal areas $(P=0.002)$. $\mathrm{CB}^{+}$neurons and processes were most prevalent in layer II (Fig. 5A$\mathrm{C}$, green; Fig. 5D and E), where they were closely associated with the distal segments of pyramidal neurons (Fig. 5F), consistent with previous studies in other cortices (e.g. DeFelipe et al., 1989a; Barbas
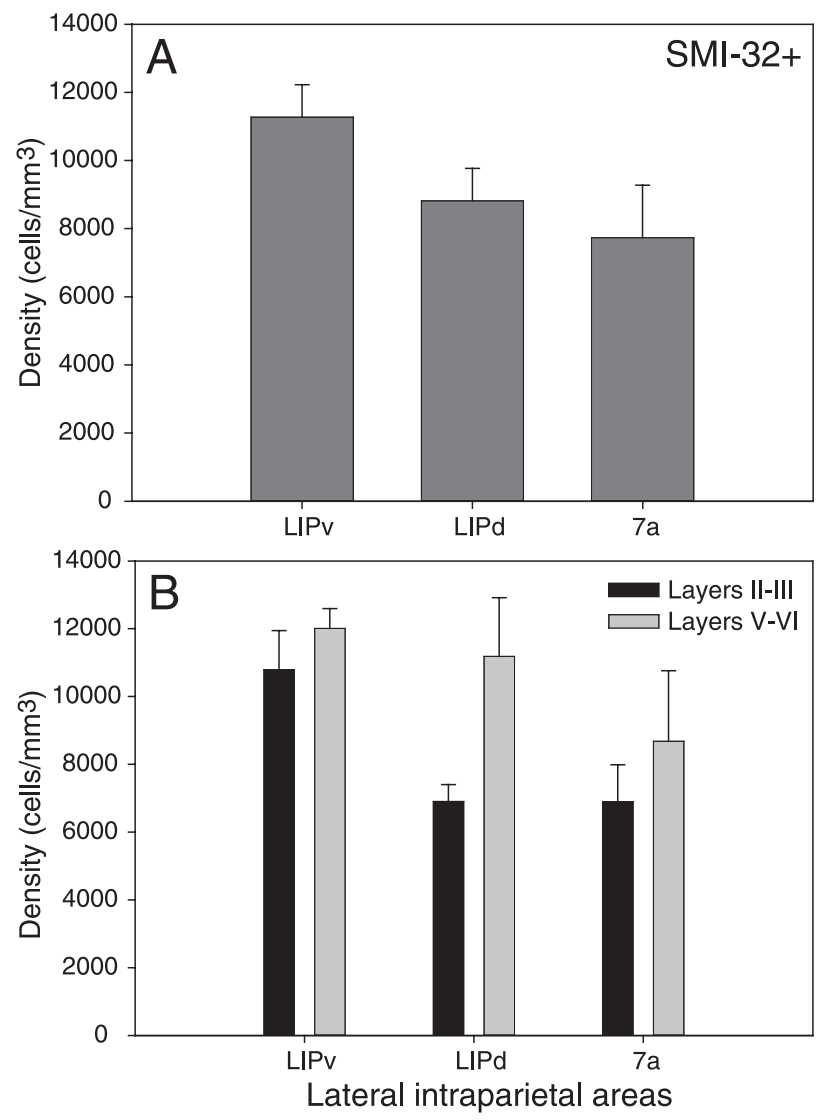

FIG. 3. Density of SMI-32 $2^{+}$neurons in lateral intraparietal areas. (A) Regional density; (B) laminar density.

et al., 2005b). $\mathrm{PV}^{+}$neurons were predominantly found in layers III-IV (Fig. 5A-C, red), where they were closely apposed to the cell bodies of pyramidal neurons (Fig. 5G; subsequent panels in Fig. 5 will be discussed later), as noted in other cortical areas (e.g. DeFelipe et al., 1989b; Lund \& Lewis, 1993; Dhar et al., 2001; Krimer \& GoldmanRakic, 2001; Barbas et al., 2005b). The global and laminar densities of $\mathrm{CB}^{+}$and $\mathrm{PV}^{+}$neurons combined did not vary across intraparietal areas (Fig. 4D and E), suggesting that inhibitory neurons vary regionally by neurochemical type but not in overall density.

\section{Connections linking periarcuate prefrontal and lateral intraparietal areas}

\section{Injection sites}

We placed bidirectional tracers in those periarcuate areas known to be connected with the visuomotor intraparietal cortex situated in the caudal half of the lateral intraparietal cortex (reviewed in Barbas, 1992). Data were obtained from four rhesus monkeys and five distinct injection sites: one in the caudal part of ventral area 46 (v46; Fig. 6A, case $\mathrm{MBH}$ ) and four sites in distinct rostrocaudal parts of area 8 . In the latter, the most rostral injection was located within the anterior arcuate gyrus, rostral to the upper limb of the arcuate sulcus, near the border of dorsal area 46 (r8; Fig. 6A, case BFr). Three of the injections in area 8 were in the caudal prearcuate region at the junction of the dorsal and ventral limbs of the arcuate sulcus. One of these injection sites was in the caudalmost part of the prearcuate region extending deep into the anterior bank of the arcuate sulcus and corresponding to the core frontal eye fields (core FEF, c8; Fig. 6A, case AD), as described in 

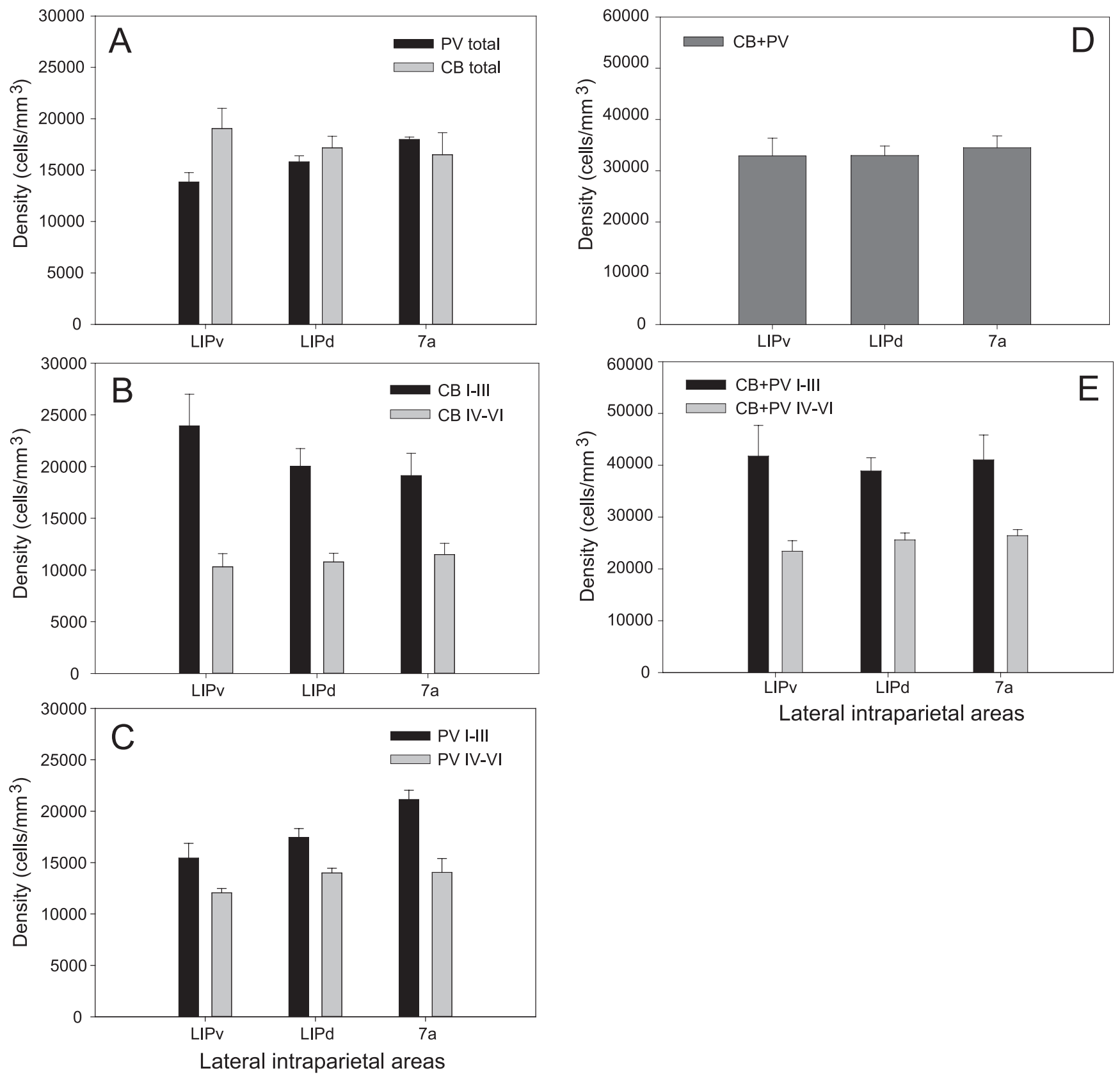

FIG. 4. Density of $\mathrm{PV}^{+}$and $\mathrm{CB}^{+}$neurons in lateral intraparietal areas. (A) Regional density of $\mathrm{PV}^{+}$(solid bars) and $\mathrm{CB}^{+}$(grey bars) neurons; (B) laminar density of $\mathrm{CB}^{+}$neurons; (C) laminar density of $\mathrm{PV}^{+}$neurons; (D) regional and (E) laminar density of $\mathrm{CB}^{+}+\mathrm{PV}^{+}$neurons combined.

previous physiologic and anatomic studies (e.g. Robinson \& Fuchs, 1969; Barbas \& Mesulam, 1981; Barbas, 1988; Schall et al., 1995; Stanton et al., 1995). The other two occupied an intermediate anteroposterior level within area 8 , near the caudal end of the principal sulcus, one more caudal (c-i8; Fig. 6A, case AC) than the other (r-i8; Fig. 6A, case $\mathrm{BFe}$ ). The $\mathrm{c}-\mathrm{i} 8$ injection (case $\mathrm{AC}$ ) covered a larger extent of the anterior arcuate gyrus than the rostral intermediate site (case $\mathrm{BFe}$ ) and was immediately anterior to the site occupied by the most caudal injection (case AD). The r-i8 injection (case BFe) was smaller than the other cases, spoon-shaped, with the head of the spoon just posterior to the principal sulcus and the narrow handle extending caudally to the arcuate gyrus and overlapping with the site of injection in case AC.

\section{Overview of connections in the lateral intraparietal areas}

Figure 6 summarizes the relative regional distribution of retrograde (Fig. 6B) and anterograde (Fig. 6C) label in the lateral intraparietal cortex, and Figs 5 and 7 show their topography on maps of the intraparietal cortex. In all cases, bidirectional label occupied approximately the middle third of the rostrocaudal extent of the lateral bank of the intraparietal sulcus. However, there were differences in the areal distribution of labelled neurons and terminations, consistent with previous findings (e.g. Barbas \& Mesulam, 1981; Barbas, 1988; Cavada \& Goldman-Rakic, 1989b; Andersen et al., 1990a; Blatt et al., 1990; Schall et al., 1995; Stanton et al., 1995; Bullier et al., 1996; Lewis \& Van Essen, 2000a). Within the above intraparietal region, 
connections of rostral periarcuate areas, v46 (case $\mathrm{MBH}$ ) and rostral area 8 (r8; case BFr), were found mostly in area 7a (Fig. 6B and C, $\mathrm{v} 46$ and $\mathrm{r} 8$ ), in the dorsolateral extent of the lateral intraparietal bank (see also Fig. 5H and I, red, for case BFr; Fig. 7A-D for case MBH). In contrast, connections of caudal area 8 (c8; case AD) were found predominantly in the ventromedial part of the lateral intraparietal

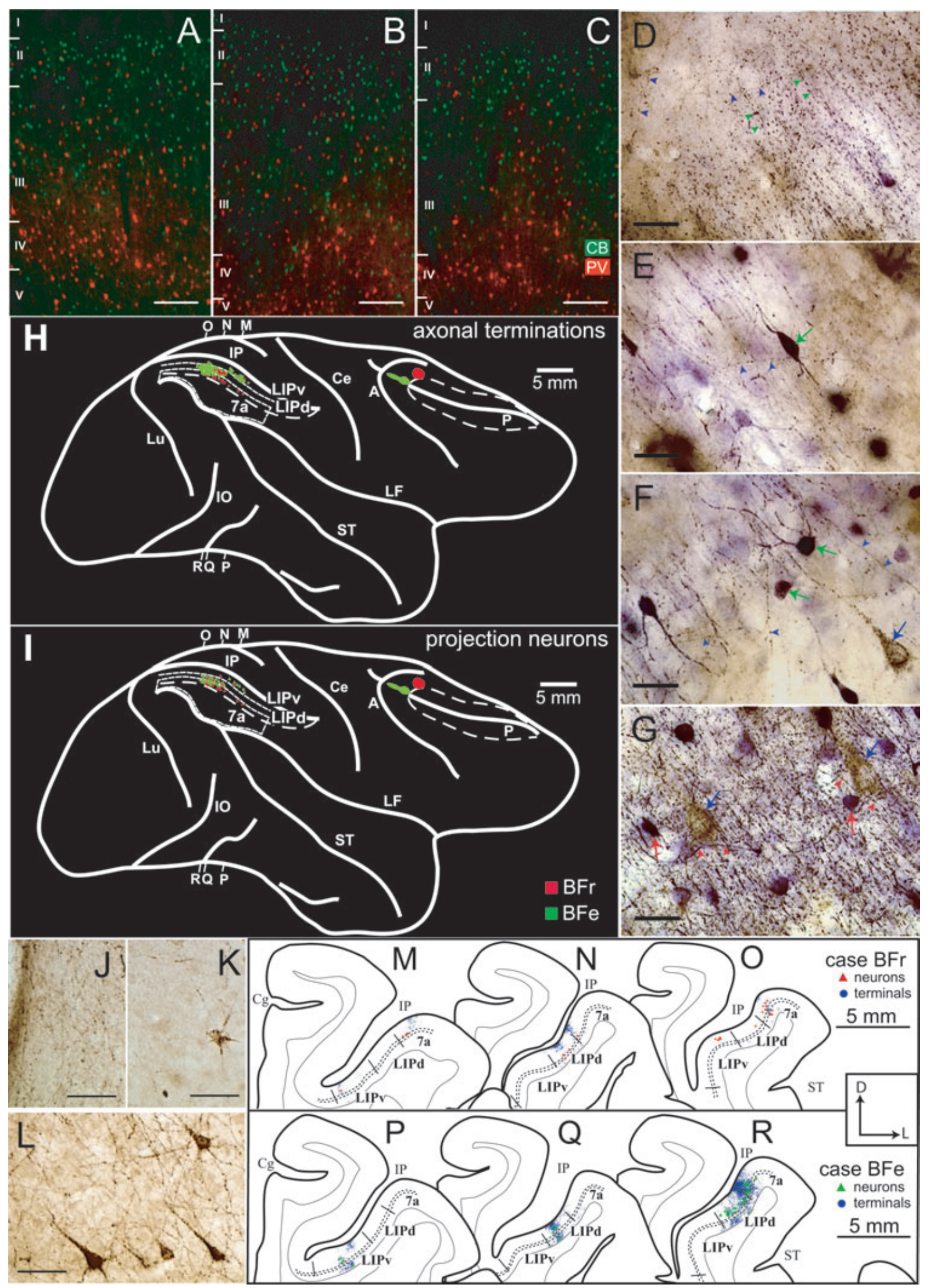

(c) The Authors (2006). Journal Compilation @ Federation of European Neuroscience Societies and Blackwell Publishing Ltd European Journal of Neuroscience, 23, 161-179 

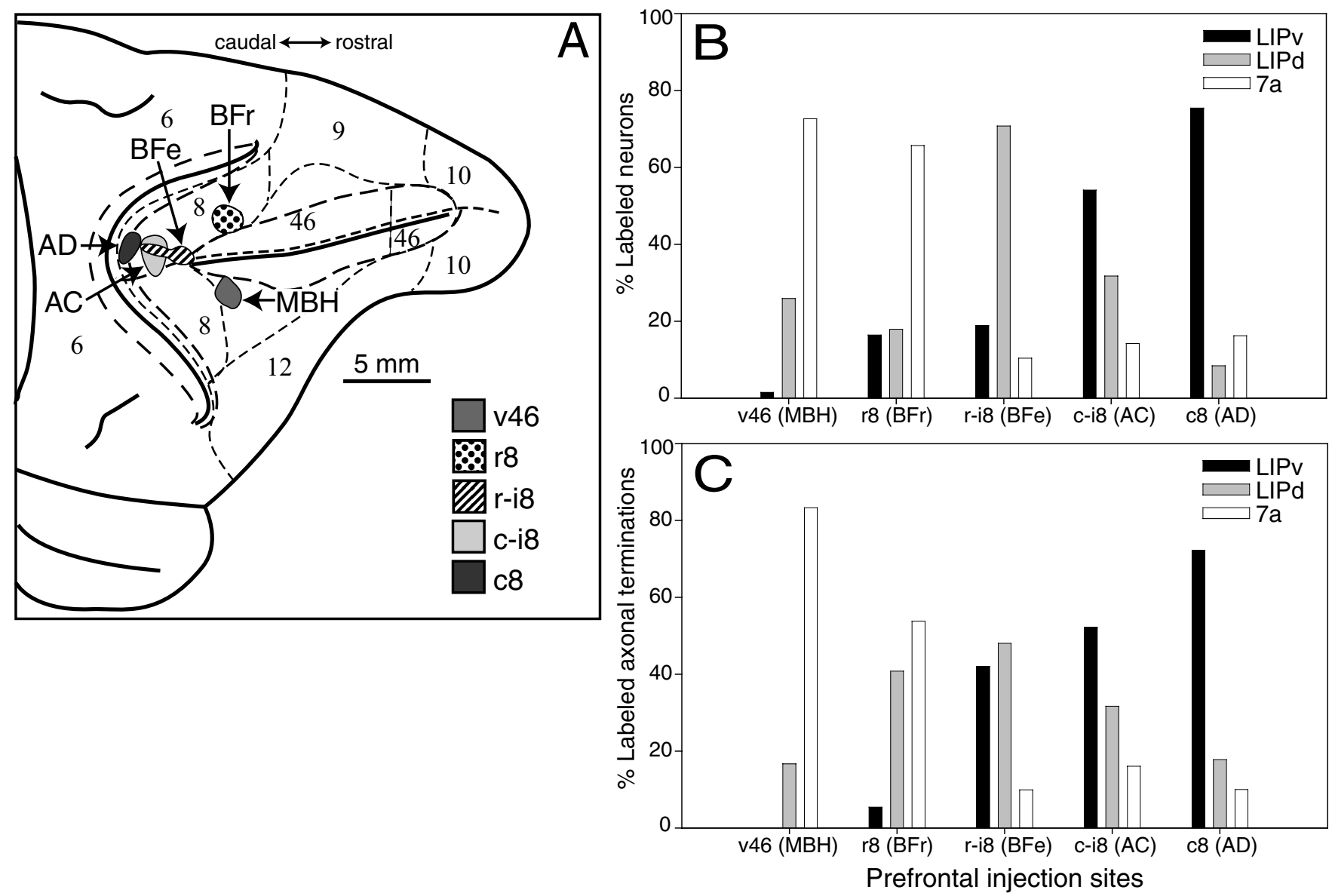

FIG. 6. Injection sites in periarcuate prefrontal areas and the topography of the resultant connections in lateral intraparietal cortex. (A) Lateral view of the prefrontal cortex showing the injection sites in areas 46 and 8 (see also Fig. 9). Short dashes mark areal boundaries; long dashes indicate shoulders of sulci. (B) Normalized regional distribution of labelled neurons in lateral intraparietal areas LIPv, LIPd and 7a. (C) Normalized regional distribution of labelled axonal terminations.

cortex, in area LIPv (Fig. 6B and C, c8; Fig. 7E-H). Connections of intermediate area 8 (cases $\mathrm{BFe}$ and $\mathrm{AC}$ ) were found mostly in areas LIPv and LIPd, in a region approximately amid the connection sites of rostral periarcuate areas and $\mathrm{c} 8$ (Fig. 6B and C, r-i8 and c-i8). There was a gradual decrease of label in area $7 \mathrm{a}$ and a concomitant increase in areas LIPd and LIPv, reflecting serially the predominant connectional associations of areas 46, r8, intermediate 8 and $\mathrm{c} 8$, as elaborated below.

\section{Connections of area 46 and $r 8$}

Areas v46 (case MBH) and r8 (case BFr) had strong connections with area $7 \mathrm{a}$ and to a lesser extent with area LIPd, and relatively weak connections with area LIPv (Fig. 6B and C, v46 and r8). The majority of labelled neurons directed to $\mathrm{r} 8$ were found in area $7 \mathrm{a}(66 \%$ of total neurons; Fig. 5K, M and O, red triangles; Fig. 6B, r8), which also included about half of the axonal terminations (54\% of total; Fig. 5J, brown grain; Fig. 5M and O, blue dots; Fig. 6C, r8). Area LIPd issued relatively weak projections to $\mathrm{r} 8(18 \%$ of total) but received comparatively stronger afferent terminals $(41 \%$ of total; Fig. $5 \mathrm{~N}$, blue dots).

The distributions of labelled neurons in intraparietal areas directed to areas 46 and rostral 8 were similar (Fig. 6B, v46 and r8). However, the proportion of terminations in area $7 \mathrm{a}$ from area 46 was relatively higher $(83 \%$ of total) than the terminations from rostral 8 (Fig. 6C,

FIG. 5. The distribution of $\mathrm{CB}^{+}$and $\mathrm{PV}^{+}$neurons, and the connections of rostral and intermediate area 8 in lateral intraparietal areas. (A) Photomicrographs of coronal sections treated for immunofluorescence showing $\mathrm{CB}^{+}$neurons (green) found mostly in layer II of area LIPv. PV neurons (red) were most prevalent in layers III-IV. A similar laminar distribution was found in (B) LIPd and (C) 7a. (D and E) Coronal sections double-stained for FE label (brown) and CB (purple) in area LIPd, under brightfield illumination. $\mathrm{CB}^{+}$processes (green arrowheads) and neurons (green arrows) are intermingled with $\mathrm{FE}^{+}$axon terminals (blue arrowheads) in layers I-II; and (F) with the distal dendrites of $\mathrm{FE}^{+}$neurons (blue arrow) in layers II-III; (G) tissue double-stained for FE (brown) and PV (purple) in area LIPd. $\mathrm{PV}^{+}$processes (red arrowheads) and neurons (red arrows) are closely apposed to soma of $\mathrm{FE}^{+}$neurons (blue arrows) in layer III. (H,I) Lateral brain surface showing topography of $(\mathrm{H})$ prefrontal terminals (each dot $=10$ boutons) and (I) labelled neurons (each triangle $=5$ neurons) in intraparietal cortex, from tracer injections in rostral (case BFr, red) and rostral intermediate (case BFe, green) area 8. Short dashes mark areal boundaries; long dashes indicate shoulders of sulci. The rostrocaudal locations of the coronal sections in M-R are shown on the lateral surface. A, arcuate sulcus; Ce, central sulcus; IO, inferior occipital sulcus; IP, intraparietal sulcus; LF lateral fissure; Lu, lunate sulcus; P, principal sulcus; ST, superior temporal sulcus. (J) Photomicrograph of $\mathrm{FR}^{+}$fibers and boutons (case $\mathrm{BFr}$ ) in layer I of area 7a; (K) labelled neuron in layer III of area 7a (case BFr); (L) FE fibers, boutons and labelled neurons in layer III of area LIPd (case BFe); (M-O) Diagrams of coronal sections showing the distribution of connections of $\mathrm{r} 8$. (P-R) Distribution of connections of r-i8. In M-R, rostral sections are to the left, caudal to the right. Dotted lines mark the boundaries of layer IV. D, dorsal; L, lateral axes. Scale bars, $150 \mu \mathrm{m}(\mathrm{A}-\mathrm{C}) ; 25 \mu \mathrm{m}(\mathrm{D}-\mathrm{G}) ; 50 \mu \mathrm{m}(\mathrm{J}-\mathrm{L})$. 

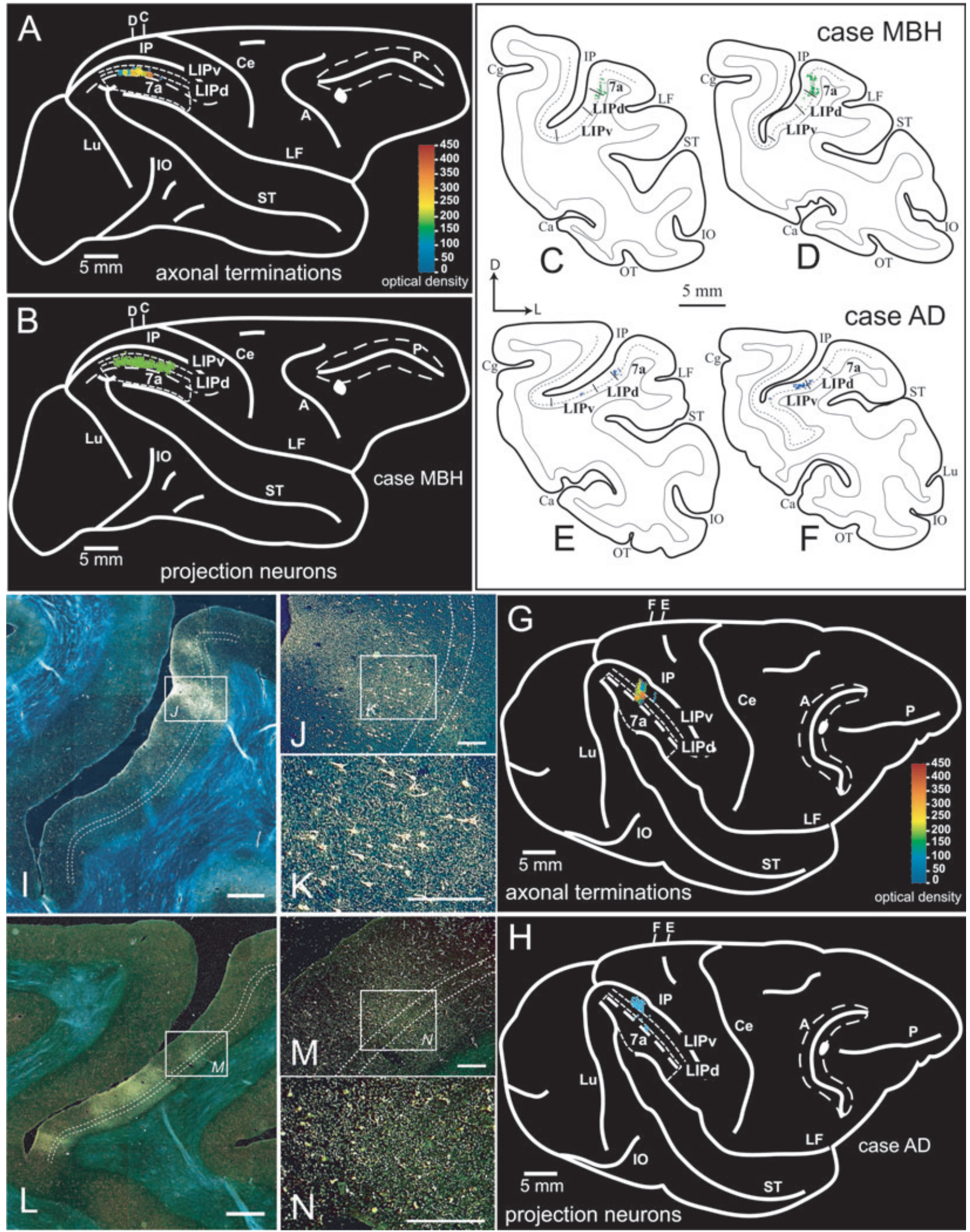
v46 and r8, silhouette bars). Axons from area 46 terminated narrowly, but reciprocal projection neurons originated from a large part of the lateral intraparietal cortex (Fig. 7A-D, case MBH).

\section{Connections of intermediate area 8}

The two intermediate area 8 sites (r-i8 and c-i8; cases BFe and AC) had a somewhat different regional distribution of intraparietal connections than areas 46 and rostral 8 . In both cases, the weakest label was noted in area 7a (Fig. 6B and C, r-i8 and c-i8). However, the two intermediate area 8 sites differed in the rostrocaudal extent and location of the injection site, with only a small region of overlap, and also showed variation in the pattern of their connections with areas LIPd and LIPv. In the case with the more rostral injection (case $\mathrm{BFe}$ ), labelling was stronger in area LIPd (neurons, 71\%; terminations, 48\%) than in area LIPv (neurons, 19\%; terminations, 42\%; Fig. 5L, labelled neurons and boutons in LIPd; Fig. 5P-R; Fig. 6B and $\mathrm{C}, \mathrm{r}-\mathrm{i} 8$ ), while the opposite was found in the case with the more caudal injection (case AC; Fig. 6B and C, c-i8; neurons, 32\%, terminations, $32 \%$ for LIPd; neurons, $54 \%$, terminations, $52 \%$ for LIPv). Moreover, the topography of connections of $\mathrm{r}-\mathrm{i} 8$ varied along the rostrocaudal extent of the intraparietal sulcus (Fig. $5 \mathrm{H}$ and I, green). Anteriorly, connections were densest with LIPv (Fig. 5P) while posteriorly, label progressively shifted to areas $7 \mathrm{a}$ and LIPd (Fig. 5Q and R).

\section{Connections of $c 8$}

Area $\mathrm{c} 8$ (case AD) differed from all other cases by its predominant connections with area LIPv (neurons, 75\%; terminations, 72\%) and to a lesser extent with areas LIPd (neurons, $8 \%$; terminations, $18 \%$ ) and 7a (neurons, 16\%; terminations, 10\%; Fig. 6B and C, c8). Moreover, the connections of $\mathrm{c} 8$ were more focal, covering a narrow rostrocaudal extent of the intraparietal region in comparison with the other cases (Fig. 7E-H, case AD).

\section{Specificity of laminar connections linking prefrontal and lateral intraparietal areas}

Consistent with the topography of connections, for progressively more caudal prefrontal sites the proportion of axonal terminations in layer I decreased, and in layer IV increased in all intraparietal areas with significant label (boutons $>50$, optical density $>$ background level). This relationship is seen in Fig. 8, where rostral periarcuate areas are shown on the top and progressively more caudal periarcuate areas are shown on the bottom. Thus, axons from the rostrally situated periarcuate prefrontal areas (areas 46 and rostral 8, and to a lesser extent intermediate area 8) terminated mostly in the superficial layers (layer I to the top part of layer III) of intraparietal cortex (Fig. 8A, C and E). In contrast, axonal terminations from c8 were comparatively weaker in the upper layers of intraparietal cortex and denser in the middle to deep layers (bottom part of layer III to top part of layer V; Fig. $8 \mathrm{G}$ and I) than for rostral periarcuate areas.
Specifically, axons from areas 46 (Fig. 7I-K, yellow grain) and r8 (Fig. 5J, brown grain; Fig. 5M-O, blue dots) terminated heavily in layer I to the top part of layer III in all intraparietal areas, especially in areas LIPd (Fig. 8A and C) and LIPv (Fig. 8C). The proportion of reciprocal projection neurons in layers II-III directed to areas 46 and r8, predominated in area LIPv (87\% in layers II-III; Fig. $8 \mathrm{~B}$, black bars) but was lower in areas LIPd (60\%; Fig. $8 \mathrm{~B}$, grey bars) and $7 \mathrm{a}$ (65\%; Fig. $8 \mathrm{~B}$ and D, silhouette bars), with the rest found in infragranular layers.

As in areas 46 and $r 8$, the connections of intermediate area 8 were densest in the upper layers of areas LIPv, LIPd and, to a lesser extent, area $7 \mathrm{a}$ (Fig. $8 \mathrm{E}-\mathrm{H})$. However, the proportion of axonal terminations from intermediate area 8 (cases $\mathrm{BFe}$ and $\mathrm{AC}$ ) was comparatively lower than for area 46 and $\mathrm{r} 8$, in the upper layers (54-80\% in layers Itop of III; compare Fig. $8 \mathrm{E}$ and $\mathrm{G}$ with Fig. $8 \mathrm{~A}$ and $\mathrm{C}$ ). A further distinction was noted between the two intermediate sites: axonal terminations from the more rostral of the two were densest in layer I (case BFe; Fig. 8E), whereas axons from the more caudal of the two terminated in a columnar pattern, with a slight bias for layer II to the top part of layer III (case AC; Fig. 8G).

Intraparietal terminations from $\mathrm{c} 8$ (core FEF, case AD) were comparatively less dense in the upper layers (30-59\% in layers I-top of III; Fig. 8I), especially layer I, than for areas 46 and r8. Moreover, the laminar pattern of connections varied across intraparietal areas. In area LIPv, axons from c8 terminated mostly in the superficial layers (59\% in layers I-top of III) but in area $7 \mathrm{a}$ and, to a lesser extent, area LIPd terminations were densest in the middle to deep layers (area 7a, 70\%; area LIPd, 62\% in layers IV-VI; Fig. 8I). Patches of anterograde label were noted specifically in layer IV of areas LIPd and $7 \mathrm{a}$, and label was weakest in layer I (Fig. 7L-N, yellow grain; Fig. 9C-E, black arrows). The reciprocal projection neurons from area $\mathrm{LIPv}$ were also predominantly found in the superficial layers, while neurons from areas LIPd and 7a were equally distributed in the deep and superficial layers (Fig. 8J).

A comparison of the relative termination of axonal fibers in intraparietal cortex from distinct prefrontal areas is seen in Fig. 9. This 3-D reference map of the lateral bank of the intraparietal sulcus summarizes and contrasts the topography and laminar terminations from a rostral case (v46, case $\mathrm{MBH}$, red), intermediate (r-i8, case BFe, green) and caudal (c8, case AD, blue) prefrontal areas (Fig. 9A-D). In Fig. 9E, the surface of layer I was removed to show the differential axonal terminations of $\mathrm{c} 8$ (case AD), encompassing all layers of area LIPv (yellow arrows), but shifting to the middle to deep layers in areas LIPd and 7a (black arrows).

\section{Relationship of laminar architecture to prefrontal connections in lateral intraparietal areas}

To determine how the lateral intraparietal areas differ in terms of structure and connection patterns, we conducted a multidimensional analysis (NMDS) using either normalized laminar architecture or laminar connectivity profiles as parameters for pairwise correlations.

FIG. 7. Connections of prefrontal area 46 and c8 in lateral intraparietal areas. (A) Lateral surface of the brain showing the topography of labelled terminals (optical density is represented in pseudocolour); and (B) labelled neurons (each dot $=5$ neurons) after injection of HRP-WGA in v46 (case MBH). Short dashes mark areal boundaries; long dashes indicate shoulders of sulci; ' $\mathrm{C}$ ' and ' $\mathrm{D}$ ' mark the rostrocaudal locations of coronal sections. (C-F) Coronal sections showing sites with label in intraparietal cortex. Distribution of projection neurons directed to (C and D, green dots) v46 (case $\mathrm{MBH}$ ) and (E and F, blue dots) c8 (case AD). In C-F, rostral sections are to the left, caudal to the right. Dotted lines mark the top of layer IV. D, dorsal; L, lateral axes. (G,H) Lateral view of the brain showing (G) anterograde label and $(\mathrm{H})$ retrograde label after injection of HRP-WGA in $\mathrm{c} 8$; ' $\mathrm{E}$ ' and ' $\mathrm{F}$ ' mark the rostrocaudal locations of coronal sections. A, arcuate sulcus; Ca, calcarine sulcus; Ce, central sulcus; Cg, cingulate sulcus; IO, inferior occipital sulcus; IP, intraparietal sulcus; LF lateral fissure; Lu, lunate sulcus; OT, occipitotemporal sulcus; P, principal sulcus; ST, superior temporal sulcus. (I) Darkfield photomicrograph of coronal section showing connections of area v46 with area 7a (case MBH). Dotted lines mark the boundaries of layer IV. (J) Columnar pattern of anterograde label in area 7a at higher magnification; (K) inset in J, magnified. (L) Darkfield photomicrograph of coronal section showing connections of c8 with areas LIPv and LIPd (case AD); (M) patch of labelled terminations in the middle layers of LIPd at higher magnification; $(\mathrm{N})$ inset in M, magnified. Scale bars: $1 \mathrm{~mm}(\mathrm{I}$ and L); $250 \mu \mathrm{m}(\mathrm{J}, \mathrm{K}, \mathrm{M}$ and N). 

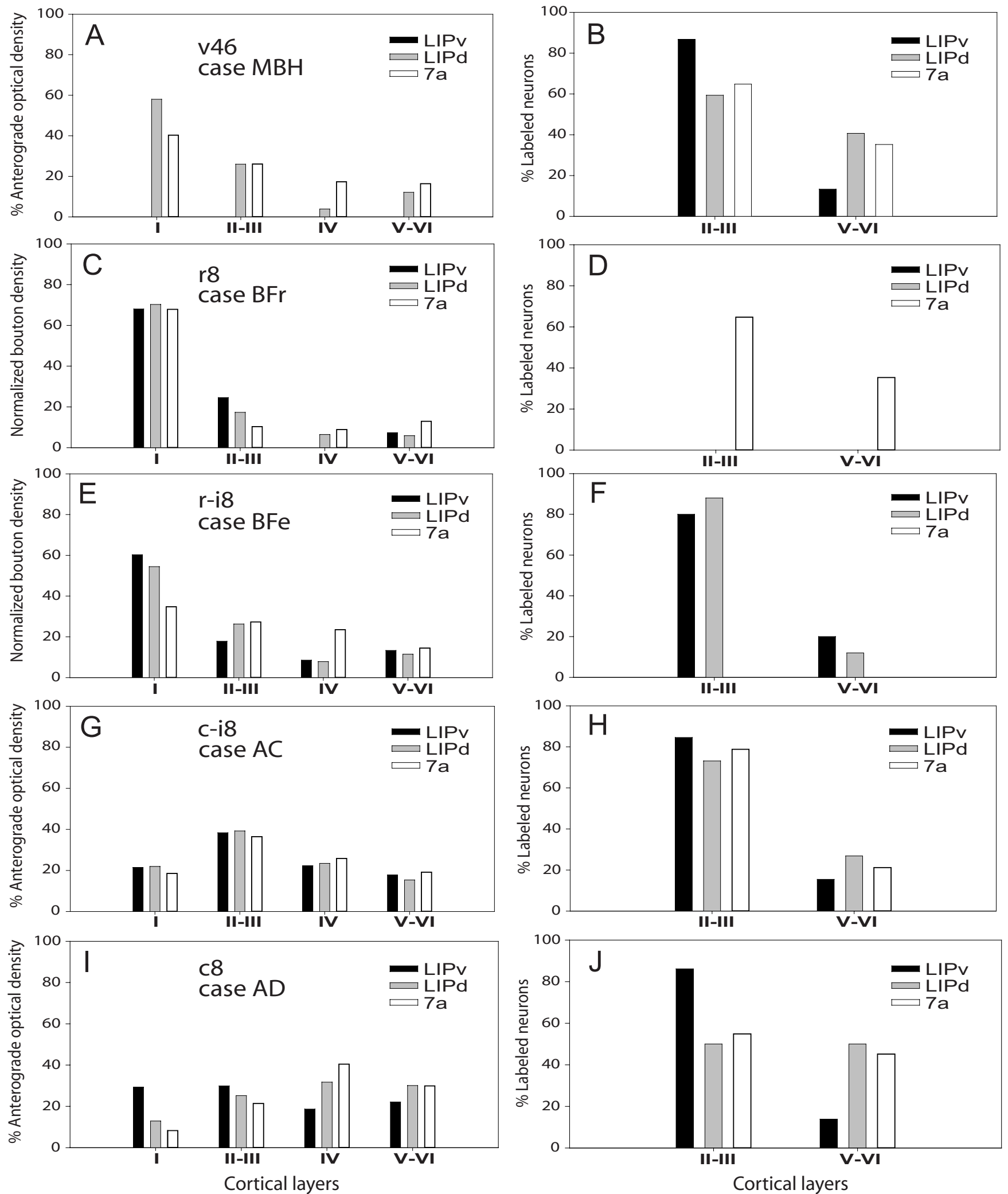

FIG. 8. Laminar distribution of prefrontal connections in lateral intraparietal cortex. Bar graphs show the normalized density of labelled axonal terminations (left) and projection neurons (right) and are arranged for tracer injections in rostral (top row) to progressively caudal (bottom row) periarcuate areas. (A and B) Injection of HRP-WGA tracer was in v46 (caudal part, case MBH). (C and D) Injection of FR tracer was in r8 (r8, case BFr). (E and F) Injection of FE tracer was in r-i8 (r-i8, case $\mathrm{BFe}$ ). ( $\mathrm{G}$ and $\mathrm{H}$ ) Injection of HRP-WGA tracer was in c-i8 (c-i8, case AC). (I and J) Injection of HRP-WGA tracer was in the caudalmost part of area 8 (core $\mathrm{FEF}, \mathrm{c} 8$, case $\mathrm{AD})$. 


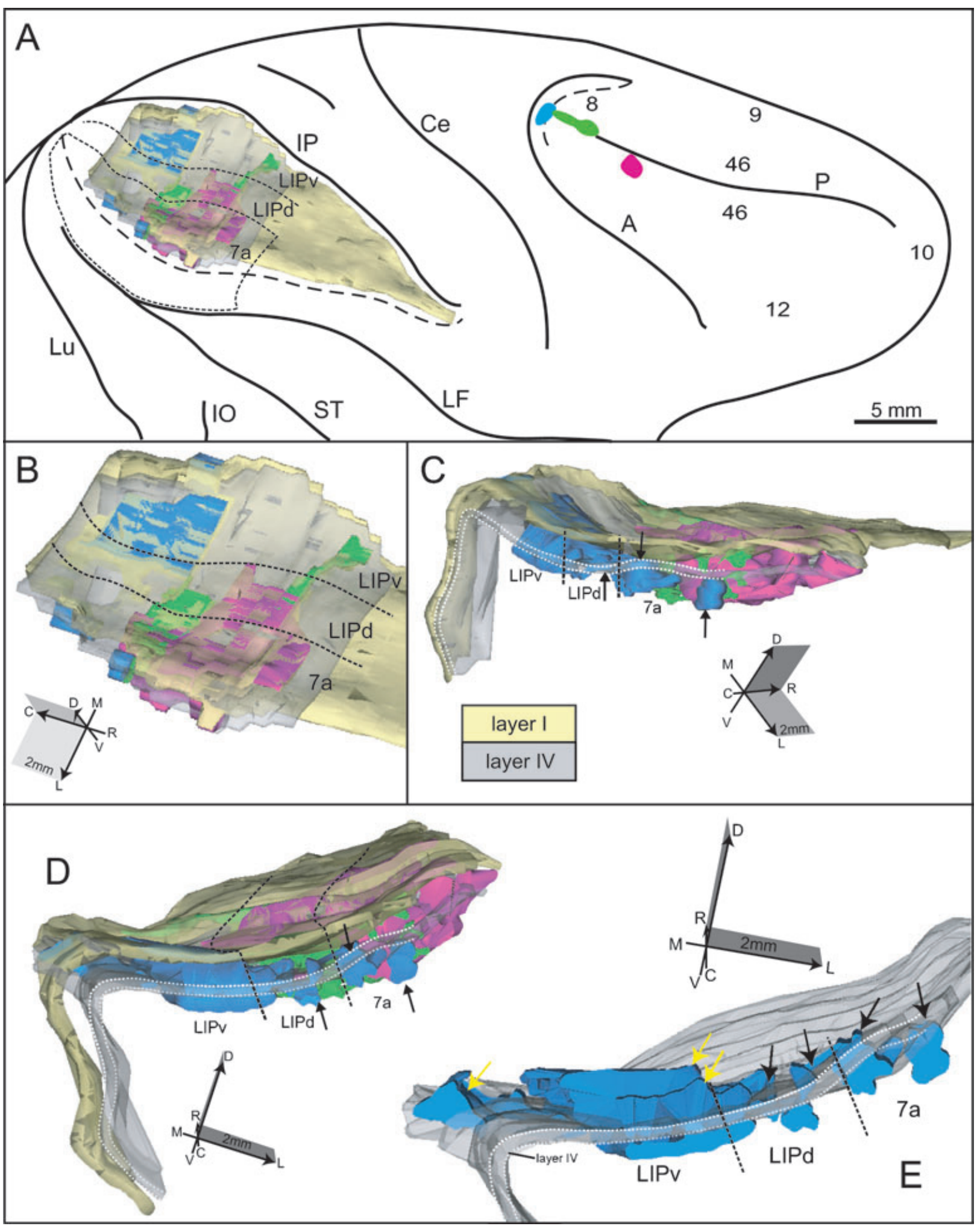

FIG. 9. 3-D reconstruction of the lateral intraparietal bank, superimposing connections of areas 46 (case MBH, red), intermediate 8 (case BFe, green) and c8 (case $\mathrm{AD}$, blue). The translucent 3-D layer I surface is shown in yellow and layer IV is shown in grey. (A) Lateral brain surface showing the location of prefrontal injection sites and the 3-D map of the lateral intraparietal bank. The sulcus is unfolded to reveal buried cortex. Long dashes indicate shoulders of sulci; short dashes delineate intraparietal areas LIPv, LIPd and 7a. Ce, central sulcus; IO, inferior occipital sulcus; IP, intraparietal sulcus; LF lateral fissure; Lu, lunate sulcus; P, principal sulcus; ST, superior temporal sulcus. (B) 3-D map from A, magnified, showing a view orthogonal to the pial surface, with anterograde label in layer I. (C) 3-D surface rotated to show a caudolateral view. (D) Caudal view showing axonal terminations in the middle layers of areas LIPd and 7a (black arrows). White dotted lines show the boundaries of layer IV with layers III and V, traced on the most caudal coronal section. (E) Caudal view showing connections of c8, with layer I surface removed to expose translucent 3-D layer IV surface (grey). Patches of label in area LIPv (blue, yellow arrows) span all layers. Label in areas LIPd and 7a (black arrows) is concentrated in the middle to deep layers. Axes indicated by arrows are as follows: R-C, rostrocaudal; M-L, mediolateral; D-V, dorsoventral. Scale bars, $2 \times 2 \mathrm{~mm}$ plane (B-E). 

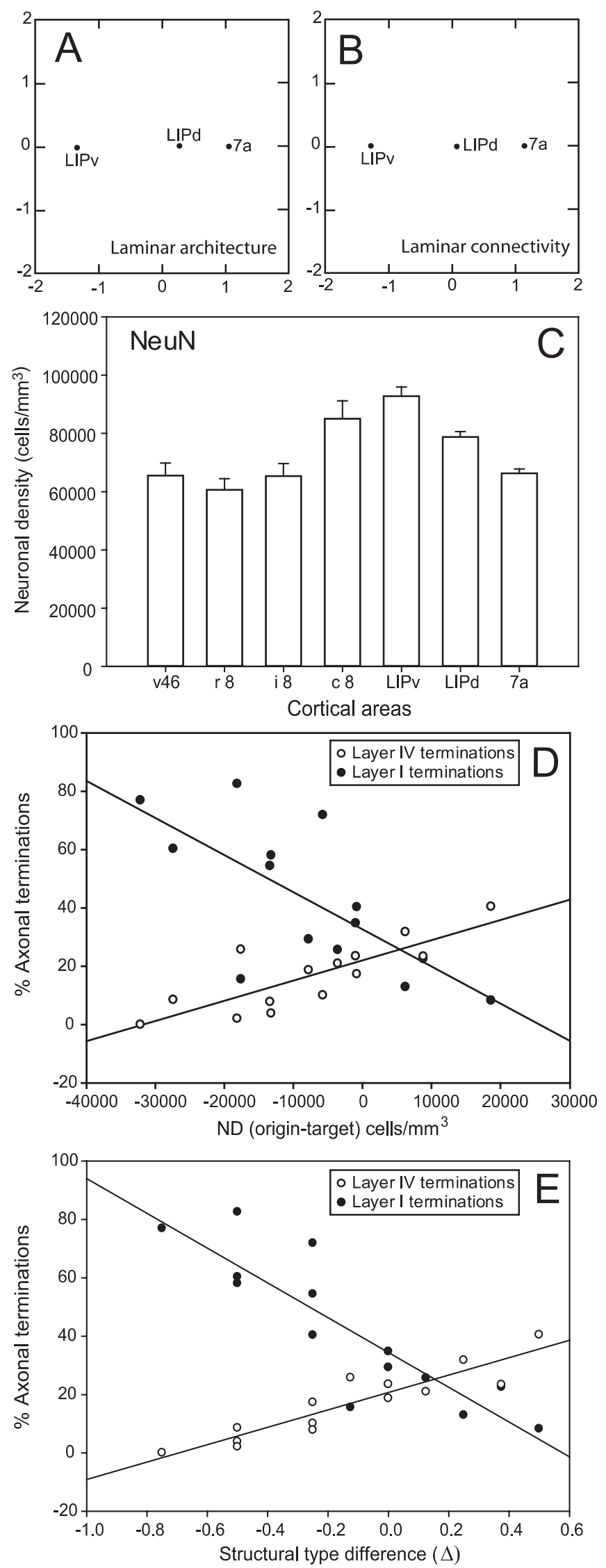

The correlation coefficients were high (Pearson's $r=0.86-0.97$, $P<0.02$ ), suggesting that the three intraparietal areas were relatively similar to each other. However, NMDS analysis showed that areas LIPd and 7a resembled each other more than area LIPv. This pattern was found for both laminar architecture (Fig. 10A) and connectivity profiles (Fig. 10B), suggesting a close relationship between architectonic parameters and the laminar pattern of connections among lateral intraparietal cortices.

We then investigated whether the structural relationship of interconnected periarcuate and intraparietal areas is related to their laminar connection patterns. A model proposed in previous studies accurately predicted that the laminar distribution of projection neurons and terminations varies according to the ordinal structural type difference $(\Delta)$ between interconnected areas (Barbas \& RempelClower, 1997; Rempel-Clower \& Barbas, 2000; Barbas et al., 2005a; for reviews see Barbas et al., 2002; Barbas \& Hilgetag, 2002). In the present study, all prefrontal and intraparietal areas are eulaminate, with six layers, belonging to structural type 5. However, these areas differed in overall neuronal density $(P=0.0009)$, consistent with findings that cortical laminar structure varies in a graded pattern (Barbas \& Pandya, 1989). Previous findings indicated that the best architectonic discriminant among prefrontal areas is neuronal density (Dombrowski et al., 2001). We thus tested whether neuronal density alone could account for the laminar pattern of connections. Figure 10C shows the overall density of neurons positive for $\mathrm{NeuN}$ in intraparietal areas and prefrontal areas matched to the respective injection sites. We found a linear relationship between the laminar proportion of terminations and the neuronal density (ND) difference between prefrontal origin and intraparietal target areas $\left(\mathrm{ND}_{\text {origin }}-\mathrm{ND}_{\text {target }}\right)$. The neuronal density difference between pairs of linked areas was positively correlated with the proportion of terminations in layer IV of intraparietal areas (Pearson's $r=0.81, P=0.0004$ ) and negatively correlated with terminations in layer I $(r=-0.71, P=0.004$; Fig. 10D). Thus, when the prefrontal origin was increasingly denser in neurons than the intraparietal target $\left(\mathrm{ND}_{\text {origin }}>\mathrm{ND}_{\text {target }}\right)$, axonal terminations increased in layer IV and concomitantly decreased in layer I. The opposite was found when the prefrontal origin had a lower neuronal density than the target $\left(\mathrm{ND}_{\text {origin }}<\mathrm{ND}_{\text {target }}\right)$, so that axonal terminations increased in layer I. If the neuronal density difference was close to zero, with the origin and target being approximately of equal density $\left(\mathrm{ND}_{\text {origin }}=\mathrm{ND}_{\text {target }}\right)$, the proportions of terminations in layer I and in layer IV were similar, resulting in an approximate columnar pattern. There was also a significant correlation with neuronal density difference for the reciprocal connections: when the neuronal density of intraparietal origin was higher than the prefrontal target, the proportion of prefrontally directed projection neurons in layers II-III was higher, with a concomitant decrease in layers $\mathrm{V}-\mathrm{VI}$ $(r=0.67, P=0.02)$.

We repeated the analysis by assigning a rating to each prefrontal and intraparietal area based on the structural model, using a finer scale to distinguish the areas as subtypes, as described in Materials and

FIG. 10. Relationship between laminar architecture and connectivity profiles of lateral intraparietal areas. (A) NMDS plot of lateral intraparietal areas using laminar architectonic parameters; (B) NMDS plot using laminar connectivity profiles produced the same clustering scheme as the architecture. (C) Neuronal density (marked by NeuN) in prefrontal and lateral intraparietal areas. (D) Relationship between neuronal density (ND) difference (between prefrontal origin and intraparietal target) and the percentage of terminations in layer IV $(\mathrm{O}, r=0.81, P=0.0004)$ and layer I $(\boldsymbol{O}, r=-0.71, P=0.0042)$ of intraparietal areas. (E) Relationship between ordinal structural type difference $(\Delta)$ and the percentage of terminations in layer IV $(\bigcirc, r=0.91, P=0.0002)$ and layer I $(\bullet, r=-0.87, P=0005)$. 
Methods. The ordinal structural type difference $(\Delta)$ between prefrontal and intraparietal areas predicted the laminar pattern of their connections $(r=0.91, P=0.0002$ for layer IV; $r=-0.87, P=0.0005$ for layer I; Fig. 10E), as did neuronal density.

\section{Relationship of connections to $\mathrm{PV}^{+}$and $\mathrm{CB}^{+}$neurons}

The highly ordered prefrontal-intraparietal pathways originated and terminated in intraparietal areas within laminar microenvironments which differed significantly in the density of the neurochemically identified classes of inhibitory interneurons labelled with PV and CB (Fig. 5A-C). Prefrontal axons, mainly from rostral periarcuate areas (areas v46 and $\mathrm{r} 8$ ) and to a lesser extent intermediate area 8 , that terminated in the upper layers of intraparietal cortex (Fig. 8A, C and E), were intermingled with $\mathrm{CB}^{+}$neurons prevalent in layer II (Fig. 5D and E). In contrast, prefrontal axons that terminated in the middle layers, originating mainly from caudal parts of area 8 (Fig. 8G and I), were intermingled with $\mathrm{PV}^{+}$neurons predominant in layers III-IV (Fig. 5G). Moreover, the increased proportion of axons from $\mathrm{c} 8$ terminating in the middle layers of areas LIPd and 7a (Fig. 8I, grey and silhouette bars) coincided with an overall increase in density of $\mathrm{PV}^{+}$neurons in these intraparietal areas (Fig. 4A, PV). In area LIPv, where axons from $\mathrm{c} 8$ predominantly terminated in the upper layers (Fig. 8I, black bars), the density of $\mathrm{PV}^{+}$neurons was comparatively lower, with a concomitant increase in the density of $\mathrm{CB}^{+}$neurons in the upper layers (Fig. 4A and B).

\section{Discussion}

The connectional relationship of distinct periarcuate prefrontal areas varied within intraparietal cortex in topography and laminar organization. Previous findings have emphasized that prefrontal-parietal connections are bilaminar (e.g. Andersen et al., 1990a; Schall et al., 1995; Stanton et al., 1995) and assumed that prefrontal cortex issues 'feedback' projections to parietal cortex, based on a hierarchical scheme of cortical processing (for review see Felleman \& Van Essen, 1991). Previous studies, however, were based either on qualitative or global quantitative data for connections. Here, using quantitative data and fine analyses for connections and architecture, we found that the core FEF, c8, targets the middle layers of intraparietal areas LIPd and $7 \mathrm{a}$, akin to 'feedforward' projections in sensory areas. Moreover, laminar connection patterns in intraparietal cortex varied consistently and predictably according to quantitative structural differences between linked prefrontal and intraparietal areas. The systematic quantitative analyses of connections, as well as cortical architecture, made it possible to discern the anatomic pattern of communication in a complex cortical association network involving areas with similar functions. These findings provide the anatomic basis to investigate the complex functional interactions of specific prefrontal-intraparietal pathways.

\section{The topography of connections is related to the structural architecture of the linked areas}

The lateral intraparietal cortex is composed of distinct architectonic areas (e.g. Seltzer \& Pandya, 1980; Andersen et al., 1985; Lewis \& Van Essen, 2000b). Here we showed that the architectonic differences can be objectively described by the density of $\mathrm{NeuN}^{+}$and SMI- $32^{+}$ neurons, which is highest in area LIPv, lower in area LIPd and lowest in area $7 \mathrm{a}$. The combined density of $\mathrm{CB}^{+}$and $\mathrm{PV}^{+}$neurons was similar across intraparietal areas, suggesting that the differences in neuronal density (NeuN) are not due to regional variations of these combined classes of inhibitory interneurons. The neuronal density differences probably reflect, at least in part, the proportion of SMI- $32^{+}$neurons, which are thought to comprise a class of long-distance projection neurons (Campbell et al., 1991; Hof et al., 1995).

Our findings showed that the topography of prefrontal connections in intraparietal areas is highly ordered, varying consistently according to the rostrocaudal position of the periarcuate areas, and is correlated with neuronal density. Rostrally situated periarcuate areas (area 46 and r8), which have lower cell densities than $\mathrm{c} 8$, were strongly associated with parietal area $7 \mathrm{a}$, which has a lower cell density than area LIPd and more so than area LIPv. Moreover, the graded changes in the topography of connections coincided with a progressive shift in functional organization: strongly interconnected periarcuate and intraparietal areas have functionally related inputs. Periarcuate and lateral intraparietal areas are associated with visuomotor and spatial functions (e.g. Wurtz \& Mohler, 1976; Lynch et al., 1977; Robinson \& Goldberg, 1978; Petrides \& Iversen, 1979; Schiller et al., 1979; Posner et al., 1984; Sakata et al., 1985; Andersen et al., 1987; Funahashi et al., 1989; Colby et al., 1996; Levy \& Goldman-Rakic, 1999; for reviews see Lynch, 1980; Tehovnik et al., 2000). However, these areas differ in the strength and organization of visual input they receive (e.g. Barbas, 1988; Cavada \& Goldman-Rakic, 1989a; Blatt et al., 1990; Stanton et al., 1995; Lewis \& Van Essen, 2000a). Areas 46, r8 and 7a, for example, have multimodal functions and receive comparatively weaker projections from visual cortices than c8 and area LIPv (Hyvärinen \& Poranen, 1974; Mesulam et al., 1977; Barbas \& Mesulam, 1981; Barbas \& Mesulam, 1985; Cavada \& GoldmanRakic, 1989a; Neal et al., 1990). In contrast, the heavily linked c8 and area $L I P v$ receive robust projections from overlapping visual association areas (Seltzer \& Pandya, 1980; Barbas \& Mesulam, 1981; Petrides \& Pandya, 1984; Barbas, 1988; Andersen et al., 1990a; Blatt et al., 1990; Baizer et al., 1991; Schall et al., 1995; Stanton et al., 1995; Bullier et al., 1996; Lewis \& Van Essen, 2000a).

The predominant connections of intermediate and $\mathrm{c} 8$ were serially organized in areas LIPd and LIPv. The core FEF (c8), which is involved in generating low-threshold saccades (Robinson \& Fuchs, 1969; Mohler et al., 1973; Bushnell et al., 1981; Bruce et al., 1985), was more strongly associated with area LIPv whereas the cortex just anterior to the core FEF (intermediate area 8) had stronger connections with area LIPd. Moreover, even small differences in the injection site, such as between the two intermediate area 8 sites (cases BFe and $\mathrm{AC}$ ), were reflected in their connections. In the more rostral intermediate area 8 site (r-i8; case $\mathrm{BFe}$ ), connections were predominant with area LIPd. On the other hand, c-i8 (case AC) showed an increase of connections with area LIPv and a concomitant decrease in area LIPd by comparison with the rostral intermediate site. This graded shift in the topography of connections coincides with progressive changes in structure along the rostrocaudal axis of the periarcuate prefrontal cortex, matched for each injection here, in parallel with similar changes seen along the lateromedial axis of intraparietal cortex (area 7a to LIPv).

\section{Neuronal density predicts the laminar pattern of connections}

The present findings provided new evidence that the structure of linked areas can be used to determine the relative distribution of efferent and afferent connections in cortical layers, consistent with the structural model for laminar connections (Barbas \& Rempel-Clower, 1997; Rempel-Clower \& Barbas, 2000). Moreover, the present data revealed that the structural model holds even for areas with small 
differences in structure, identified objectively by their neuronal density (see also Barbas et al., 2005a). For example, the proportion of terminations in layer IV was higher when the neuronal density of the prefrontal origin was higher than the intraparietal destination. Conversely, the proportion of terminations in layer I was higher, with a concomitant decrease in terminations in layer IV, when the neuronal density of prefrontal origin was lower than the target. These findings provide novel evidence that the simple and objective measure of neuronal density difference can reliably predict the laminar distribution of connections as well as an ordinal rating system (e.g. Barbas \& Rempel-Clower, 1997). These findings may form the basis for understanding the complex functional dialogue between prefrontal and intraparietal areas. More generally, these findings suggest that neuronal density may be used to infer the pattern of connections in the human cortex where architecture, but not connectivity, can be readily studied.

\section{Implications on prefrontal-parietal functional interactions}

It has been suggested that prefrontal cortex mediates 'top-down' mechanisms for recognition and selection of relevant stimuli, by influencing activity in earlier-processing cortical areas (e.g. Thompson \& Schall, 2000; Gehring \& Knight, 2002; Freedman et al., 2003). Evidence from sensory systems has led to the assumption that information processing in the cortex occurs in a hierarchical manner (for review see Felleman \& Van Essen, 1991). However, the hierarchical scheme may not account for the interactions between cortical association areas of similar function, such as periarcuate prefrontal and lateral intraparietal cortices, which are activated in parallel during complex oculomotor tasks (e.g. Lynch, 1992; Tian \& Lynch, 1996; Chafee \& Goldman-Rakic, 2000; Wurtz et al., 2001). Laminar-specific connections originate and terminate within laminar microenvironments that differ substantially (for review see Barbas et al., 2002) and thus may provide insight on how distinct areas interact. Our results show that the periarcuate cortex does not only issue 'feedback' signals to intraparietal areas as previously assumed but also targets the middle layers, analogous to 'feedforward' connections in sensory systems. Thus, the diverse laminar relationships of prefrontal-intraparietal interconnections may underlie the functional diversity of these cortices. Prefrontal input thus may exert different influences, modulating intraparietal activity through projections to layer I as well as driving activity via connections to the middle layers (see also Germuska et al., 2005).

Prefrontal axons that terminate in the upper layers probably contact the distal apical dendrites of pyramidal neurons (for reviews see Rockland, 1994; Elston, 2002; Elston, 2003) and may gradually enhance their outputs (e.g. Shao \& Burkhalter, 1996). Axonal terminations in layers I-II were highly intermingled with $\mathrm{CB}^{+}$ neurons, which were more abundant in the upper layers; as has been noted in other cortical areas (e.g. DeFelipe et al., 1989a; Hendry et al., 1989; Condé et al., 1994; Gabbott \& Bacon, 1996; Glezer et al., 1998; Kondo et al., 1999; Dombrowski et al., 2001; Barbas et al., 2005b). Thus, prefrontal axons terminating in the upper layers may activate $\mathrm{CB}^{+}$neurons, which are thought to be involved in tuning preferred responses by suppressing nonpreferred cues according to a theoretical model of working memory (Wang et al., 2004). In this context, it is interesting that in area LIPv a high proportion of prefrontal axons from area 8 terminating in the upper layers coincided with a high proportion of $\mathrm{CB}^{+}$neurons and a high density of output neurons (SMI$32^{+}$and labelled projection neurons). This suggests a mechanism for tuning activity in area $\mathrm{LIPv}$ related to the selection and maintenance of task-relevant targets during spatial allocation of attention (Ben Hamed \& Duhamel, 2002).

On the other hand, projections from $\mathrm{c} 8$ (core FEF) directed to areas LIPd and 7a predominantly terminated in the middle layers, which receive input from early processing visual areas and the thalamus (Jones \& Burton, 1976; Lewis \& Van Essen, 2000a; for reviews see Salin \& Bullier, 1995; Jones, 1998) and issue corticocortical projections (for review see Nowak \& Bullier, 1997). Moreover, prefrontal axonal terminations in the middle layers were intermingled with $\mathrm{PV}^{+}$inhibitory neurons, which exert strong and localized inhibition, as seen in other areas (e.g. Shao \& Burkhalter, 1996; Rao et al., 1999; Gonzalez-Burgos et al., 2000; Krimer \& GoldmanRakic, 2001). Thus, the core FEF can directly influence the inputs and outputs of areas LIPd and 7a by rapidly driving excitation or inhibition, possibly to convey appropriate preparatory signals for 'filling-in' of information, especially when external sensory information is ambiguous. Previous findings show that neurons in LIP respond to stimuli brought by a saccade to their receptive fields, even before the saccade occurs and stimuli enter the receptive fields (Duhamel et al., 1992; Quaia et al., 1998). Recent evidence indicates that LIP neurons fire when the perceived direction of a motion stimulus is towards their receptive fields, even when motion is ambiguous (Shadlen \& Newsome, 2001; Williams et al., 2003). Thus, it is possible that $\mathrm{c} 8$, which receives early and detailed visual input (Barbas \& Mesulam, 1981; Barbas, 1988; Schall et al., 1995; Thompson \& Schall, 2000), drives activity in LIPd and 7a through 'feedforward' connections targeting the middle layers. This pathway may convey an early, yet untuned, representation of the stimulus associated with an initial behavioural decision or saccade plan.

Neurons in area LIPd fire tonically during active fixation, suggesting a role in attentional processing and fine-grain analysis of the environment (Ben Hamed \& Duhamel, 2002; Ben Hamed et al., 2002). Neurons in area $7 \mathrm{a}$ have been implicated in complex spatial tasks involving sensory integration and have large and bilateral receptive fields, occurring within head-centred coordinates (e.g. Mesulam et al., 1977; Motter \& Mountcastle, 1981; Andersen et al., 1990b; Blatt et al., 1990; Brotchie et al., 1995; Constantinidis \& Steinmetz, 2001a,b; Crowe et al., 2004). However, when gaze or attention is shifted during fine-grain and complex analysis, driving signals from core FEF to the middle layers of areas LIPd and 7a may have a role in anticipating the shift to disengage from fixation and redirect attentional resources. Prefrontal areas, including FEF, show significantly earlier visual stimulus-driven activity than some highorder visual association areas (Thompson \& Schall, 2000; for reviews see Nowak \& Bullier, 1997; Bar, 2003). There is evidence that prefrontal cortex may generate such anticipatory signals, based on generalized rules, to categorize and specify task-relevant information in posterior cortical areas (Quintana \& Fuster, 1993; Freedman et al., 2001; Shulman et al., 2002; Freedman et al., 2003; for reviews see Miller et al., 2002; Bar, 2003). The present findings provide the connectional basis for a similar mechanism based on the specific interactions between core FEF and the middle layers of intraparietal areas LIPd and 7a.

The functional complexity of prefrontal-intraparietal interactions may be consequent to diverse laminar structural and connectional relationships, such that different microcircuits and pathways within the network may be engaged depending on the timing and nature of the task at hand. The laminar circuitry presented here suggests that the frontal eye field is capable of conveying an early, possibly predictive, decision signal to distinct intraparietal areas that may initiate not only purposive eye movements, but also complex cognitive processes such as working memory, attention and perception. 


\section{Acknowledgements}

We thank Dr Claus Hilgetag for help with the NMDS analysis, Dr John Fiala for help with the 3-D reconstruction and Ms. Ola Alade for excellent technical assistance. Supported by NIH grants from NINDS and NIMH.

\section{Abbreviations}

7a, parietal area 7a (or PG) in the caudal inferior parietal lobule; $\mathrm{c} 8$, caudal area $8 ; \mathrm{CB}$, calbindin; c-i8, caudal intermediate area 8 ; FE, fluoroemerald; FEF, frontal eye field; FR, fluororuby; HRP-WGA, horseradish peroxidase conjugated to wheatgerm agglutinin; LIP, lateral intraparietal area (or area POa); LIPd or area POa-e, dorsal (or external) division of LIP; LIPv or POa-i, ventral (or internal) division of LIP; ND, neuronal density; NeuN, neuronal nucleispecific antibody; NGS, normal goat serum; NMDS, nonparametric multidimensional scaling; PB, phosphate buffer; PBS, phosphate-buffered saline; PV, parvalbumin; r8, rostral area 8; r-i8, rostral intermediate area 8; SMI-32, antibody of nonphosphorylated neurofilament H protein; v46, ventral area 46.

\section{References}

Andersen, R.A., Asanuma, C. \& Cowan, W.M. (1985) Callosal and prefrontal associational projecting cell populations in area $7 \mathrm{a}$ of the macaque monkey: a study using retrogradely transported fluorescent dyes. J. Comp. Neurol., 232, 443-455.

Andersen, R.A., Asanuma, C., Essick, G. \& Siegel, R.M. (1990a) Corticocortical connections of anatomically and physiologically defined subdivisions within the inferior parietal lobe. J. Comp. Neurol., 296, 65-113.

Andersen, R.A., Bracewell, R.M., Barash, S., Gnadt, J.W. \& Fogassi, L. (1990b) Eye position effects on visual, memory, and saccade-related activity in areas LIP and 7a of macaque. J. Neurosci., 10, 1176-1196.

Andersen, R.A., Essick, G.K. \& Siegel, R.M. (1987) Neurons of area 7 activated by both visual stimuli and oculomotor behavior. Exp. Brain Res., 67, 316-322.

Baizer, J.S., Ungerleider, L.G. \& Desimone, R. (1991) Organization of visual inputs to the inferior temporal and posterior parietal cortex in macaques. J. Comp. Neurol., 11, 168-190.

Bar, M. (2003) A cortical mechanism for triggering top-down facilitation in visual object recognition. J. Cogn. Neurosci., 15, 600-609.

Barbas, H. (1988) Anatomic organization of basoventral and mediodorsal visual recipient prefrontal regions in the rhesus monkey. J. Comp. Neurol., 276, 313-342.

Barbas, H. (1992) Architecture and cortical connections of the prefrontal cortex in the rhesus monkey. Adv. Neurol., 57, 91-115.

Barbas, H. \& De Olmos, J. (1990) Projections from the amygdala to basoventral and mediodorsal prefrontal regions in the rhesus monkey. J. Comp. Neurol., 301, 1-23.

Barbas, H., Ghashghaei, H., Dombrowski, S.M. \& Rempel-Clower, N.L. (1999) Medial prefrontal cortices are unified by common connections with superior temporal cortices and distinguished by input from memory-related areas in the rhesus monkey. J. Comp. Neurol., 410, 343-367.

Barbas, H., Ghashghaei, H., Rempel-Clower, N. \& Xiao, D. (2002) Anatomic basis of functional specialization in prefrontal cortices in primates. In Grafman, J. (Ed.), Handbook of Neuropsychology. Elsevier Science BV, Amsterdam, pp. 1-27.

Barbas, H. \& Hilgetag, C.C. (2002) Rules relating connections to cortical structure in primate prefrontal cortex. Neurocomputing, 44-46, 301-308.

Barbas, H., Hilgetag, C.C., Saha, S., Dermon, C.R. \& Suski, J.L. (2005a) Parallel organization of contralateral and ipsilateral prefrontal cortical projections in the rhesus monkey. BMC Neurosci., 6, 32.

Barbas, H., Medalla, M., Alade, O., Suski, J., Zikopoulos, B. \& Lera, P. (2005b) Relationship of prefrontal connections to inhibitory systems in superior temporal areas in the rhesus monkey. Cereb. Cortex, 15, 13561370.

Barbas, H. \& Mesulam, M.M. (1981) Organization of afferent input to subdivisions of area 8 in the rhesus monkey. J. Comp. Neurol., 200, 407-431.

Barbas, H. \& Mesulam, M.M. (1985) Cortical afferent input to the principalis region of the rhesus monkey. Neuroscience, 15, 619-637.

Barbas, H. \& Pandya, D.N. (1987) Architecture and frontal cortical connections of the premotor cortex (area 6) in the rhesus monkey. J. Comp. Neurol., 256, 211-218.

Barbas, H. \& Pandya, D.N. (1989) Architecture and intrinsic connections of the prefrontal cortex in the rhesus monkey. J. Comp. Neurol., 286, 353-375.
Barbas, H. \& Rempel-Clower, N. (1997) Cortical structure predicts the pattern of corticocortical connections. Cereb. Cortex, 7, 635-646.

Ben Hamed, S. \& Duhamel, J.R. (2002) Ocular fixation and visual activity in the monkey lateral intraparietal area. Exp. Brain Res., 142, 512-528.

Ben Hamed, S., Duhamel, J.R., Bremmer, F. \& Graf, W. (2002) Visual receptive field modulation in the lateral intraparietal area during attentive fixation and free gaze. Cereb. Cortex, 12, 234-245.

Blatt, G.J., Andersen, R.A. \& Stoner, G.R. (1990) Visual receptive field organization and cortico-cortical connections of the lateral intraparietal area (Area LIP) in the Macaque. J. Comp. Neurol., 299, 421-445.

Brotchie, P.R., Andersen, R.A., Snyder, L.H. \& Goodman, S.J. (1995) Head position signals used by parietal neurons to encode locations of visual stimuli. Nature, 375, 232-235.

Bruce, C.J. \& Goldberg, M.E. (1985) Primate frontal eye fields. I. Single neurons discharging before saccades. J. Neurophysiol., 53, 603-635.

Bruce, C.J., Goldberg, M.E., Bushnell, M.C. \& Stanton, G.B. (1985) Primate frontal eye fields. II. Physiological and anatomical correlates of electrically evoked eye movements. J. Neurophysiol., 54, 714-734.

Bullier, J., Schall, J.D. \& Morel, A. (1996) Functional streams in occipitofrontal connections in the monkey. Behav. Brain Res., 76, 89-97.

Bushnell, M.C., Goldberg, M.E. \& Robinson, D.L. (1981) Behavioral enhancement of visual responses in monkey cerebral cortex. I. Modulation in posterior parietal cortex related to selective visual attention. J. Neurophysiol., 46, 755-772.

Campbell, M.J., Hof, P.R. \& Morrison, J.H. (1991) A subpopulation of primate corticocortical neurons is distinguished by somatodendritic distribution of neurofilament protein. Brain Res., 539, 133-136.

Cavada, C. \& Goldman-Rakic, P.S. (1989a) Posterior parietal cortex in rhesus monkey. I. Parcellation of areas based on distinctive limbic and sensory corticocortical connections. J. Comp. Neurol., 287, 393-421.

Cavada, C. \& Goldman-Rakic, P.S. (1989b) Posterior parietal cortex in rhesus monkey. II. Evidence for segregated corticocortical networks linking sensory and limbic areas with the frontal lobe. J. Comp. Neurol., 287, 422-445.

Chafee, M.V. \& Goldman-Rakic, P.S. (1998) Matching patterns of activity in primate prefrontal area $8 \mathrm{a}$ and parietal area 7 ip neurons during a spatial working memory task. J. Neurophysiol., 79, 2919-2940.

Chafee, M.V. \& Goldman-Rakic, P.S. (2000) Inactivation of parietal and prefrontal cortex reveals interdependence of neural activity during memoryguided saccades. J. Neurophysiol., 83, 1550-1566.

Colby, C.L., Duhamel, J.R. \& Goldberg, M.E. (1996) Visual, presaccadic, and cognitive activation of single neurons in monkey lateral intraparietal area. J. Neurophysiol., 76, 2841-2852.

Colby, C.L. \& Goldberg, M.E. (1999) Space and attention in parietal cortex. Annu. Rev. Neurosci., 22, 319-349.

Condé, F., Lund, J.S., Jacobowitz, D.M., Baimbridge, K.G. \& Lewis, D.A. (1994) Local circuit neurons immunoreactive for calretinin, calbindin D-28k or parvalbumin in monkey prefrontal cortex: distribution and morphology. J. Comp. Neurol., 341, 95-116.

Constantinidis, C. \& Steinmetz, M.A. (2001a) Neuronal responses in area 7a to multiple-stimulus displays: I. neurons encode the location of the salient stimulus. Cereb. Cortex, 11, 581-591.

Constantinidis, C. \& Steinmetz, M.A. (2001b) Neuronal responses in area 7a to multiple stimulus displays: II. responses are suppressed at the cued location. Cereb. Cortex, 11, 592-597.

Crowe, D.A., Chafee, M.V., Averbeck, B.B. \& Georgopoulos, A.P. (2004) Neural activity in primate parietal area 7a related to spatial analysis of visual mazes. Cereb. Cortex, 14, 23-34.

Cusick, C.G., Seltzer, B., Cola, M. \& Griggs, E. (1995) Chemoarchitectonics and corticocortical terminations within the superior temporal sulcus of the rhesus monkey: evidence for subdivisions of superior temporal polysensory cortex. J. Comp. Neurol., 360, 513-535.

DeFelipe, J., Gonzalez-Albo, M.C., del Rio, M.R. \& Elston, G.N. (1999) Distribution and patterns of connectivity of interneurons containing calbindin, calretinin, and parvalbumin in visual areas of the occipital and temporal lobes of the macaque monkey. J. Comp. Neurol., 412, 515-526.

DeFelipe, J., Hendry, S.H. \& Jones, E.G. (1989a) Synapses of double bouquet cells in monkey cerebral cortex visualized by calbindin immunoreactivity. Brain Res., 503, 49-54.

DeFelipe, J., Hendry, S.H. \& Jones, E.G. (1989b) Visualization of chandelier cell axons by parvalbumin immunoreactivity in monkey cerebral cortex. Proc. Natl. Acad. Sci. USA, 86, 2093-2097.

Dhar, P., Mehra, R.D., Sidharthan, V. \& Sharma, K. (2001) Parvalbumin and calbindin D-28K immunoreactive neurons in area MT of rhesus monkey. Exp. Brain Res., 137, 141-149. 
Dombrowski, S.M., Hilgetag, C.C. \& Barbas, H. (2001) Quantitative architecture distinguishes prefrontal cortical systems in the rhesus monkey. Cereb. Cortex, 11, 975-988.

Duhamel, J.R., Colby, C.L. \& Goldberg, M.E. (1992) The updating of the representation of visual space in parietal cortex by intended eye movements. Science, 255, 90-92.

Elston, G.N. (2002) Cortical heterogeneity: implications for visual processing and polysensory integration. J. Neurocytol., 31, 317-335.

Elston, G.N. (2003) Cortex, cognition and the cell: new insights into the pyramidal neuron and prefrontal function. Cereb. Cortex, 13, 1124-1138.

Felleman, D.J. \& Van Essen, D.C. (1991) Distributed hierarchical processing in the primate cerebral cortex. Cereb. Cortex, 1, 1-47.

Fiala, J.C. (2005) Reconstruct: a free editor for serial section microscopy. J. Microsc., 218, 52-61.

Freedman, D.J., Riesenhuber, M., Poggio, T. \& Miller, E.K. (2001) Categorical representation of visual stimuli in the primate prefrontal cortex. Science, 291, 312-316.

Freedman, D.J., Riesenhuber, M., Poggio, T. \& Miller, E.K. (2003) A comparison of primate prefrontal and inferior temporal cortices during visual categorization. J. Neurosci., 23, 5235-5246.

Funahashi, S., Bruce, C.J. \& Goldman-Rakic, P.S. (1989) Mnemonic coding of visual space in the monkey's dorsolateral prefrontal cortex. J. Neurophysiol., 61, 331-349.

Fuster, J.M. (1973) Unit activity in prefrontal cortex during delayed-response performance: neuronal correlates of transient memory. J. Neurophysiol., 36, $61-78$.

Fuster, J.M. (1984) Behavioral electrophysiology of the prefrontal cortex. Trends Neurosci., 7, 408-414.

Gabbott, P.L. \& Bacon, S.J. (1996) Local circuit neurons in the medial prefrontal cortex (areas 24a,b,c, 25 and 32) in the monkey. II. Quantitative areal and laminar distributions. J. Comp. Neurol., 364, 609-636.

Gehring, W.J. \& Knight, R.T. (2002) Lateral prefrontal damage affects processing selection but not attention switching. Brain Res. Cogn. Brain Res., 13, 267-279.

Germuska, M., Saha, S., Fiala, J. \& Barbas, H. (2005) Synaptic distinction of laminar specific prefrontal-temporal pathways in primates. Cereb. Cortex, in press.

Glezer, I.I., Hof, P.R. \& Morgane, P.J. (1998) Comparative analysis of calciumbinding protein-immunoreactive neuronal populations in the auditory and visual systems of the bottlenose dolphin (Tursiops truncatus) and the macaque monkey (Macaca fascicularis). J. Chem. Neuroanat., 15, 203-237.

Gonzalez-Burgos, G., Barrionuevo, G. \& Lewis, D.A. (2000) Horizontal synaptic connections in monkey prefrontal cortex: an in vitro electrophysiological study. Cereb. Cortex, 10, 82-92.

Gundersen, H.J.G., Bagger, P., Bendtsen, T.F., Evans, S.M., Korbo, L., Marcussen, N., Moller, A., Nielsen, K., Nyengaard, J.R., Pakkenberg, B., Sorensen, F.B., Vesterby, A. \& West, M.J. (1988) The new stereological tools: disector, fractionator, nucleator and point sample intercepts and their use in pathological research and diagnosis. APMIS, 96, 857-881.

Hendry, S.H.C., Jones, E.G., Emson, P.C., Lawson, D.E.M., Heizmann, C.W. \& Streit, P. (1989) Two classes of cortical GABA neurons defined by differential calcium binding protein immunoreactivities. Exp. Brain Res., 76, 467-472.

Hof, P.R., Nimchinsky, E.A. \& Morrison, J.H. (1995) Neurochemical phenotype of corticocortical connections in the macaque monkey: Quantitative analysis of a subset of neurofilament protein-immunoreactive projection neurons in frontal, parietal, temporal, and cingulate cortices. J. Comp. Neurol., 362, 109-133.

Howard, C.V. \& Reed, M.G. (1998) Unbiased Stereology, Three-Dimensional Measurement in Microscopy. BIOS Scientific Publishers Limited, Oxford.

Hyvärinen, J. (1982) Posterior parietal lobe of the primate brain. Physiol. Rev., 62, 1060-1129.

Hyvärinen, J. \& Poranen, A. (1974) Function of the parietal associative area 7 as revealed from cellular discharges in alert monkeys. Brain, 97, 673-692.

Jones, E.G. (1998) A new view of specific and nonspecific thalamocortical connections. Adv. Neurol., 77, 49-71.

Jones, E.G. \& Burton, H. (1976) Areal differences in the laminar distribution of thalamic afferents in cortical fields of the insular, parietal and temporal regions of primates. J. Comp. Neurol., 168, 197-248.

Kondo, H., Hashikawa, T., Tanaka, K. \& Jones, E.G. (1994) Neurochemical gradient along the monkey occipito-temporal cortical pathway. Neuroreport, 5, 613-616.

Kondo, H., Tanaka, K., Hashikawa, T. \& Jones, E.G. (1999) Neurochemical gradients along monkey sensory cortical pathways: calbindin-immunoreactive pyramidal neurons in layers II and III. Eur. J. Neurosci., 11, 4197-4203.
Krimer, L.S. \& Goldman-Rakic, P.S. (2001) Prefrontal microcircuits: membrane properties and excitatory input of local, medium, and wide arbor interneurons. J. Neurosci., 21, 3788-3796.

Kubota, K., Iwamoto, T. \& Suzuki, H. (1974) Visuokinetic activities of primate prefrontal neurons during delayed-response performance. J. Neurophysiol., 37, 1197-1212.

Levy, R. \& Goldman-Rakic, P.S. (1999) Association of storage and processing functions in the dorsolateral prefrontal cortex of the nonhuman primate. J. Neurosci., 19, 5149-5158.

Lewis, J.W. \& Van Essen, D.C. (2000a) Corticocortical connections of visual, sensorimotor, and multimodal processing areas in the parietal lobe of the macaque monkey. J. Comp. Neurol., 428, 112-137.

Lewis, J.W. \& Van Essen, D.C. (2000b) Mapping of architectonic subdivisions in the macaque monkey, with emphasis on parieto-occipital cortex. J. Comp. Neurol., 428, 79-111.

Lund, J.S. \& Lewis, D.A. (1993) Local circuit neurons of developing and mature macaque prefrontal cortex: Golgi and immunocytochemical characteristics. J. Comp. Neurol., 328, 282-312.

Lynch, J.C. (1980) The functional organization of posterior parietal association cortex. Behav. Brain Sci., 3, 485-534.

Lynch, J.C. (1992) Saccade initiation and latency deficits after combined lesions of the frontal and posterior eye fields in monkeys. J. Neurophysiol., 68, 1913-1916.

Lynch, J.C., Mountcastle, V.B., Talbot, W.H. \& Yin, T.C.T. (1977) Parietal lobe mechanisms for directed visual attention. J. Neurophysiol., 40, 362-389.

Mesulam, M.M. (1999) Spatial attention and neglect: parietal, frontal and cingulate contributions to the mental representation and attentional targeting of salient extrapersonal events. Philos. Trans. R. Soc. Lond. B. Biol. Sci., 354, 1325-1346.

Mesulam, M.M., Hegarty, E., Barbas, H., Carson, K.A., Gower, E.C., Knapp, A.G., Moss, M.B. \& Mufson, E.J. (1980) Additional factors influencing sensitivity in the tetramethyl benzidine method for horseradish peroxidase neurohistochemistry. J. Histochem. Cytochem., 28, 1255-1259.

Mesulam, M.M., Van Hoesen, G.W., Pandya, D.N. \& Geschwind, N. (1977) Limbic and sensory connections of the inferior parietal lobule (area PG) in the rhesus monkey: a study with a new method for horseradish peroxidase histochemistry. Brain Res., 136, 393-414.

Miller, E.K., Freedman, D.J. \& Wallis, J.D. (2002) The prefrontal cortex: categories, concepts and cognition. Philos. Trans. R. Soc. Lond. B. Biol. Sci., 357, 1123-1136.

Mohler, C.W., Goldberg, M.E. \& Wurtz, R.H. (1973) Visual receptive fields of frontal eye field neurons. Brain Res., 61, 385-389.

Motter, B.C. \& Mountcastle, V.B. (1981) The functional properties of the posterior parietal cortex studied in waking monkeys: Foveal sparing and opponent vector organization. J. Neurosci., 1, 3-26.

Neal, J.W., Pearson, R.C.A. \& Powell, T.P.S. (1990) The connections of area PG, 7a, with cortex in the parietal, occipital and temporal lobes of the monkey. Brain Res., 532, 249-264.

Niki, H. \& Watanabe, M. (1976) Prefrontal unit activity and delayed response: relation to cue location versus direction of response. Brain Res., 105, 79-89.

Nowak, L.G. \& Bullier, J. (1997) The timing of information transfer in the visual system. In Rockland, K.S., Kaas, J.H. \& Peters, A. (Eds), Cerebral Cortex: Extrastriate Cortex in Primates. Plenum Press, New York, pp. 205241.

Petrides, M. \& Iversen, S.D. (1979) Restricted posterior parietal lesions in the rhesus monkey and performance on visuospatial tasks. Brain Res., 161, 63-77.

Petrides, M. \& Pandya, D.N. (1984) Projections to the frontal cortex from the posterior parietal region in the rhesus monkey. J. Comp. Neurol., 228, 105116.

Posner, M.I., Walker, J.A., Friedrich, F.J. \& Rafal, R.D. (1984) Effects of parietal injury on covert orienting of attention. J. Neurosci., 4, 1863-1874.

Quaia, C., Optican, L.M. \& Goldberg, M.E. (1998) The maintenance of spatial accuracy by the perisaccadic remapping of visual receptive fields. Neural Netw., 11, 1229-1240.

Quintana, J. \& Fuster, J.M. (1993) Spatial and temporal factors in the role of prefrontal and parietal cortex in visuomotor integration. Cereb. Cortex, $\mathbf{3}$, 122-132.

Rao, S.G., Williams, G.V. \& Goldman-Rakic, P.S. (1999) Isodirectional tuning of adjacent interneurons and pyramidal cells during working memory: evidence for microcolumnar organization in PFC. J. Neurophysiol., 81, 1903-1916.

Rempel-Clower, N.L. \& Barbas, H. (1998) Topographic organization of connections between the hypothalamus and prefrontal cortex in the rhesus monkey. J. Comp. Neurol., 398, 393-419. 
Rempel-Clower, N.L. \& Barbas, H. (2000) The laminar pattern of connections between prefrontal and anterior temporal cortices in the rhesus monkey is related to cortical structure and function. Cereb. Cortex, 10, 851-865.

Robinson, D.A. \& Fuchs, A.F. (1969) Eye movements evoked by stimulation of the frontal eye fields. J. Neurophysiol., 32, 637-648.

Robinson, D.L. \& Goldberg, M.E. (1978) Sensory and behavioral properties of neurons in posterior parietal cortex of the awake, trained monkey. Fed. Proc., 37, 2258-2261.

Rockland, K.S. (1994) The organization of feedback connections from area V2 (18) to V1 (17). In Peters, A. \& Rockland, K.S. (eds), Cerebral Cortex, Vol. 10. Plenum Press, New York, pp. 261-299.

Rosene, D.L., Roy, N.J. \& Davis, B.J. (1986) A cryoprotection method that facilitates cutting frozen sections of whole monkey brains from histological and histochemical processing without freezing artifact. J. Histochem. Cytochem., 34, 1301-1315.

Sakata, H., Shibutani, H., Kawano, K. \& Harrington, T.L. (1985) Neural mechanisms of space vision in the parietal association of cortex of the monkey. Vision Res., 25, 453-463.

Salin, P.A. \& Bullier, J. (1995) Corticocortical connections in the visual system: structure and function. Physiol. Rev., 75, 107-154.

Schall, J.D., Morel, A., King, D.J. \& Bullier, J. (1995) Topography of visual cortex connections with frontal eye field in macaque: Convergence and segregation of processing streams. J. Neurosci., 15, 4464-4487.

Schiller, P.H., True, S.D. \& Conway, J.L. (1979) Effects of frontal eye field and superior colliculus ablations on eye movements. Science, 206, 590592.

Schmitz, C. \& Hof, P.R. (2005) Design-based stereology in neuroscience. Neuroscience, 130, 813-831.

Seltzer, B. \& Pandya, D.N. (1980) Converging visual and somatic sensory cortical input to the intraparietal sulcus of the rhesus monkey. Brain Res., 192, 339-351.

Shadlen, M.N. \& Newsome, W.T. (2001) Neural basis of a perceptual decision in the parietal cortex (area LIP) of the rhesus monkey. J. Neurophysiol., 86, 1916-1936.
Shao, Z. \& Burkhalter, A. (1996) Different balance of excitation and inhibition in forward and feedback circuits of rat visual cortex. J. Neurosci., 16, 73537365 .

Shibutani, H., Sakata, H. \& Hyvärinen, J. (1984) Saccade and blinking evoked by microstimulation of the posterior parietal association cortex of the monkey. Exp. Brain Res., 55, 1-8.

Shulman, G.L., d'Avossa, G., Tansy, A.P. \& Corbetta, M. (2002) Two attentional processes in the parietal lobe. Cereb. Cortex, 12, 1124-1131.

Stanton, G.B., Bruce, C.J. \& Goldberg, M.E. (1995) Topography of projections to posterior cortical areas from the macaque frontal eye fields. J. Comp. Neurol., 353, 291-305.

Tehovnik, E.J., Sommer, M.A., Chou, I.H., Slocum, W.M. \& Schiller, P.H. (2000) Eye fields in the frontal lobes of primates. Brain Res. Brain Res. Rev., 32, 413-448.

Thompson, K.G. \& Schall, J.D. (2000) Antecedents and correlates of visual detection and awareness in macaque prefrontal cortex. Vision Res., 40, 15231538.

Tian, J. \& Lynch, J.C. (1996) Corticocortical input to the smooth and saccadic eye movement subregions of the frontal eye field in Cebus monkeys. J. Neurophysiol., 76, 2754-2771.

Vogt, C. \& Vogt, O. (1919) Allgemeinere Ergebnisse unserer Hirnforschung. J. Psychol. Neurol., 25, 279-462.

Von Bonin, G. \& Bailey, P. (1947) The Neocortex of Macaca Mulatta. The University of Illinois Press, Urbana.

Wang, X.J., Tegner, J., Constantinidis, C. \& Goldman-Rakic, P.S. (2004) Division of labor among distinct subtypes of inhibitory neurons in a cortical microcircuit of working memory. Proc. Natl. Acad. Sci. USA, 101, 1368-1373.

Williams, Z.M., Elfar, J.C., Eskandar, E.N., Toth, L.J. \& Assad, J.A. (2003) Parietal activity and the perceived direction of ambiguous apparent motion. Nat. Neurosci., 6, 616-623.

Wurtz, R.H. \& Mohler, C.W. (1976) Enhancement of visual responses in monkey striate cortex and frontal eye fields. J. Neurophysiol., 39, 766-772.

Wurtz, R.H., Sommer, M.A., Pare, M. \& Ferraina, S. (2001) Signal transformations from cerebral cortex to superior colliculus for the generation of saccades. Vision Res., 41, 3399-3412. 